\title{
Multi-level transaction management for complex objects implementation, performance, parallelism
}

\section{Report}

Author(s):

Hasse, Christof; Weikum, Gerhard

Publication date:

1991

Permanent link:

https://doi.org/10.3929/ethz-a-000597283

Rights / license:

In Copyright - Non-Commercial Use Permitted

Originally published in:

ETH, Eidgenössische Technische Hochschule Zürich, Departement Informatik, Institut für Computersysteme 162 


\section{EII}

Eidgenössische

Technische Hochschule Zürich
Departement Informatik Institut für Informationssysteme
Gerhard Weikum Christof Hasse

\section{Multi-Level Transaction Management for Complex Objects: \\ Implementation, Performance, Parallelism}

July 1991

Eidg. Tehm. Ho:hschule Zürich Informatikbiblotivek

ETH-Zentrum CH-8092 Zürich 
Authors' address:

Institut für Informationssysteme ETH-Zentrum $\mathrm{CH}-8092$ Zurich, Switzerland e-mail: weikum@inf.ethz.ch

CC 1991 Departement Informatik, ETH Zürich 


\title{
Multi-Level Transaction Management for Complex Objects: Implementation, Performance, Parallelism*
}

\author{
Gerhard Weikum and Christof Hasse \\ Computer Science Department \\ ETH Zurich \\ $\mathrm{CH}-8092$ Zurich, Switzerland \\ E-Mail: \{weikum,hasse\}@inf.ethz.ch
}

\begin{abstract}
Multi-level transactions are a variant of open nested transactions in which the subtransactions correspond to operations at different levels of a layered system architecture. The point of multilevel transactions is that the semantics of high-level operations can be exploited in order to increase concurrency. As a consequence, undoing a transaction requires compensation of completed subtransactions. In addition, multi-level recovery methods have to take into account that high-level operations are not necessarily atomic if multiple pages are updated in a single subtransaction.

This paper presents algorithms for multi-level transaction management that are implemented in the database kernel system DASDBS. In particular, it is shown that multi-level recovery can be implemented in an efficient way. We discuss performance measurements, using a synthetic benchmark for processing complex objects in a multi-user environment. In addition, it is shown that multi-level transaction management can be easily extended to cope with parallel subtransactions within a single transaction. Performance results are presented with varying degrees of inter-and intra-transaction parallelism.
\end{abstract}

\section{Introduction}

Multi-level transactions are a variant of open nested transactions in which the subtransactions correspond to operations at different levels of a layered system architecture [BSW88]. The point of multi-level transactions is that the semantics of high-level operations can be exploited in order to increase concurrency. For example, two "deposit" operations on a bank account are commutative and can therefore be admitted concurrently (e.g., on behalf of two funds transfer transactions). However, executing such high-level operations in parallel requires that a low-level synchronization mechanism takes care of possible low-level conflicts, e.g., on indexes or data pages. In relational DBMSs where records do not span pages, this low-level synchronization is usually implemented by page latches, i.e., cheap semaphores that are held while a page is accessed. ** For advanced DBMSs with complex high-level operations that may access many pages in a dynamically determined (i.e., not pre-defined) order, the simple latching method is not feasible since it cannot ensure the indivisibility of arbitrary multi-page update operations. Rather, high-level operations need to be executed as subtransactions that are dealt with by a general concurrency control mechanism at the lower level. This principle, which can be applied to an arbitrary number of levels, ensures that the semantic concurrency control at the top level need not care about lower-level conflicts.

\footnotetext{
This project is supported by the Unlon Bank of Switzeriand (Schweizerische Bankgesellschaft). ** For indexes, latching is actually more complicated (see [ML89]). It is feasible because there are specific constraints on the order in which index pages are accessed during index operations.
} 
In this paper, we address multi-level transaction management in advanced DBMSs that deal with complex objects, by applying multi-level transaction management to the following two levels:

- At the object level L1, semantic locks are dynamically acquired and held until end-of-transaction (EOT) according to the strict two-phase locking protocol. The semantics of the high-level operations is exploited in the lock modes and the lock mode compatibility table, which is in turn derived from the commutativity properties or semantic compatibility [Ga83, SZ89] of the operations. In principle, one could even exploit state-dependent commutativity [O'N86, We88], but this is beyond the scope of this paper.

- At the page level Lo, page locks are dynamically acquired during the execution of a subtransaction and are released at end-of-subtransaction (EOS). Note that, unlike in conventional nested transactions [M085], the locks of a subtransaction are not inherited by the parent. Releasing the low-level locks as early as possible while retaining only a semantically richer lock at a higher level is exactly why multi-level transaction management allows more concurrency than single-level protocols.

An example of a (correct) parallel execution of two multi-level transactions is shown in Figure 1. Suppose an office document filing system where documents have a complex structure and can span many pages. Users modify documents by specific high-level operations such as 1) "change the font of all instances of a particular component type (e.g., text paragraphs)" and 2) "change the contents of a figure". These two Change operations on the same document are commutative; however, since they may access many subobjects of the document (e.g., because the layout of the entire document is recomputed), the potential conflicts at the lower level have to be dealt with. In Figure 1, this is done by acquiring locks on the underlying pages that are released at the end of the subtransactions $T_{11}, T_{12}$, and $T_{21}$, respectively.

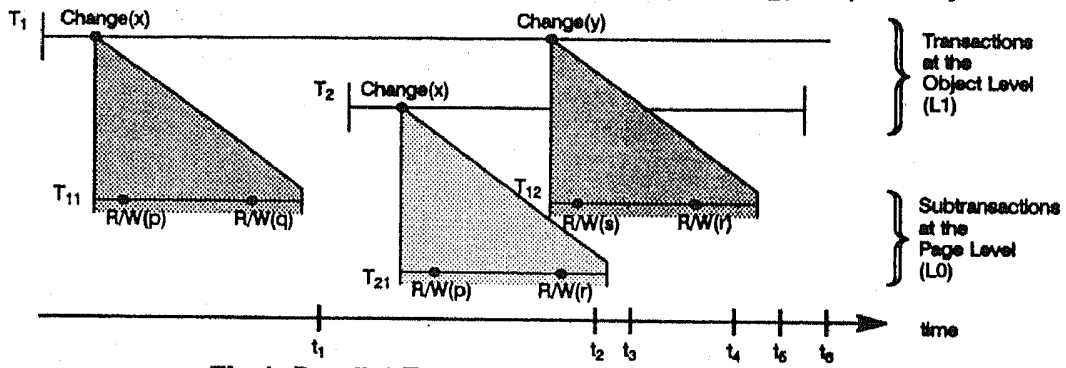

Fig.1: Parallel Execution of Two Multi-Level Transactions

Similar examples arise in advanced business applications with large amounts of derived data. For example, in foreign exchange transactions, a forward transaction (e.g., a currency swap) may have to compute a large number of future positions for risk assessment (e.g., to compute how many Japanese Yen a bank will hold at a particular date). In such an application, the potential data contention can be reduced by updating the derived data within subtransactions that release low-level locks early.

An inherent consequence of multi-level locking is that transactions can no longer be undone by simple state-oriented recovery methods at the page level. Rather, since page locks have been released at EOS, completed subtransactions must be compensated by inverse high-level operations. These operations are in turn executed as so-called compensating subtransactions [MGG86, Wei87, BSW88, GS87, KLS90, SDP91, We89, Wei91]. In the example of Figure 1, un- 
doing transaction $T_{1}$ would require two inverse Change operations on $y$ and $x$, i.e., two additional subtransactions that compensate the completed subtransactions $T_{12}$ and $T_{11}$ (reversing the order of the original subtransactions).

Compensating subtransactions are necessary for both handling transaction aborts and crash recovery after a system failure. An important prerequisite is that both regular subtransactions and compensating subtransactions have to be atomic. Otherwise, the recovery after a crash may be faced with a database state that is not sufficiently consistent to perform the necessary high-level undo steps. For example, the storage structures of a complex object may contain dangling pointers, or some derived data may only partially reflect the primary updates. If a subtransaction modifies multiple pages, as shown in Figure 1, a low-level recovery mechanism at the page level is necessary in order to provide subtransaction atomicity. This problem is challenging in that a straightforward implementation of multi-level recovery may cause excessive logging and could thus diminish the benefits of the enhanced concurrency of multi-level transactions.

Theoretical and practical issues of multi-level transaction management have been addressed by a variety of papers [BBG89, BF89, BR90, BSW88, Bü88, CF90, FLMW88, GS87, HH88, Ma87, MGG86, MR91, RGN90, SDP91, SG88, Sh85, WS84, WS91, Wei86, Wei87, WHBM90, Wei91]. However, to our knowledge, none of the previous work has presented a full implementation. Furthermore, only two papers have presented performance figures. [Wei91] contains performance measurements with a multi-level transaction manager built on top of the commercial Codasyl database system UDS; the results were strongly affected by the fact that UDS could not be changed in these experiments. [BR90] contains simulation results on multi-level concurrency control only; i.e., disregarding recovery issues.

Our paper makes the following novel contributions:

- It shows how multi-level transaction management can be efficiently implemented. The implementation is integrated in the database kernel system DASDBS [SPSW90].

- It presents performance measurements of the implemented system, based on a synthetic benchmark for complex-object processing.

- It shows how multi-level transaction management can be extended so that subtransactions of the same transaction can be executed in parallel. Performance results are presented with varying degrees of inter- and intra-transaction parallelism.

Parts of this paper have been published in [HW91]. In this highly extended paper, we discuss the implementation of multi-level recovery in much more detail, we discuss additional performance experiments, and we have added the issue of intra-transaction parallelism including preliminary performance results. The rest of the paper is organized as follows. Section 2 presents our implementation of multi-level transaction management, with emphasis on the performance-critical recovery component. Section 3 discusses the simple extensions that we have made to cope with intra-transaction parallelism. Section 4 discusses the results of a comprehensive series of performance experiments. Section 5 compares our implementation with related work, especially the ARIES recovery method [Mo89]. Section 6 discusses several options for further improving the performance of multi-level transaction management. 


\section{Implementation of Multi-Level Transaction Management in DASDBS}

\subsection{Lock Management}

Our lock manager can manage multiple lock tables that are specified to handle particular types of lockable items (e.g., pages, objects, objects of different object types, index keys, keys of different indexes, etc.). The lock manager supports a restricted form of predicate locking [EGLT76], namely conjunctive predicates of the form Attr $_{1}=$ Value $_{1}$ And $\ldots$ And Attr ${ }_{n}=$ Value $_{n}$, with $n$ being a lock table creation parameter. Efficient access to the locks that are in potential conflict with a lock request is provided through hash tables on one or more of the $n$ attributes (as specified at the creation time of the lock table). Thus, the special case of conjunctive predicate locks without wild cards can be managed fairly efficiently; and for $n=1$, our lock manager is essentially no different from conventional implementations [Gr78].

Dynamic allocation of lock control blocks is implemented by a tunable shared-memory heap manager that is optimized toward frequent disposals and reallocations of memory fragments of particular sizes. Deadlock detection is implemented by using an algorithm for partial transitive closures, which is invoked on each lock conflict. This algorithm operates on an $m^{\star} m$ waitfor matrix where $m$ is the maximum degree of multiprogramming that was specified at system startup time.

In addition to the usual lock modes "shared" and "exclusive", semantic lock modes such as "increment" can be incorporated by specifying the lock mode compatibility matrix at the creation time of a lock table [SS84]. In the performance experiments that are described in Section 4 , this feature was not exploited; rather, shared and exclusive locks were acquired on sets of object identifiers.

\subsection{Recovery Management}

This subsection contains an in-depth discussion of our implementation of multi-level recovery. The implemented algorithms are based on the methodical framework of [WHBM90]; here we give an implementation-oriented algorithmic description. In Subsection 2.2.1, we present a simple "strawman" algorithm that is based on applying the DB Cache method [EB84] to pagelevel subtransactions. The strawman algorithm provides correctness but has potential performance problems in that it may cause excessive $\log \mathrm{l} / \mathrm{Os}$. Therefore, the algorithm is refined in Subsection 2.2.2 by adding the concept of deferred log writes. Note that deferring log writes may be straightforward in a single-level recovery method with page locking, but it incurs significant problems in multi-level transaction management where multi-page update subtransactions of incomplete (i.e., uncommitted) transactions may have modified common pages. We show how our implementation solves these problems, thus saving a substantial amount of log I/Os. Finally, in Subsection 2.2.3, we discuss the idempotence problem that arises during the warmstart, and present our approach to coping with non-idempotent high-level operations.

\subsubsection{Requirements and Overall Approach}

A method for multi-level recovery must satisfy the following requirements:

1) It must ensure that transactions are atomic.

2) It must ensure that transactions are persistent.

3) It must ensure that subtransactions are atomic.

Note that subtransactions need not be persistent before the commitment of their parent. In addition to the above requirements for correctness, the following performance requirement is 
reasonable in order to guarantee an acceptable recovery time and hence high availability of the DBMS:

4) During a warmstart, redo (of committed transactions) should be performed at the bottom level Lo, i.e., by reconstructing pages rather than reexecuting potentially resource-intensive high-level operations.

An architecture that meets the above requirements is shown in Figure 2. For requirement 1 , undo log records are written at the object level L1. Each of these log records contains information about the compensating subtransaction that is necessary to undo an executed high-level operation. The log records of a transaction are chained together in a backward chain for handling transaction aborts and for performing transaction undo after a crash. In addition to the $L 1$ operation log records, EOT log records are written for completed (i.e., committed or aborted) transactions.

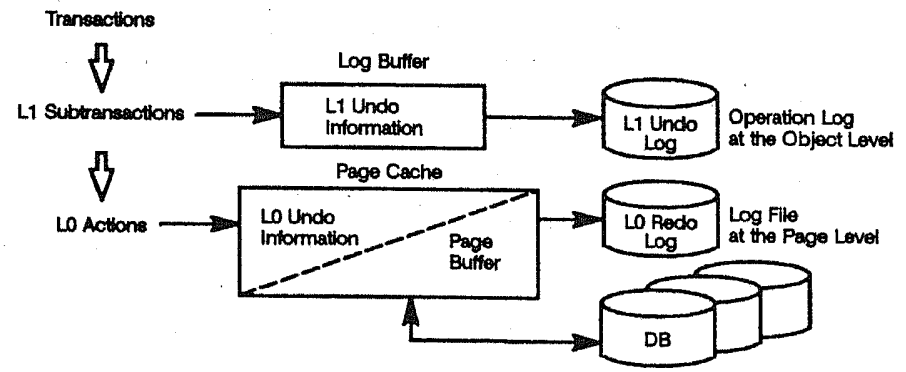

Fig.2: Architecture of the DASDBS Multi-Level Transaction Management

For requirements 2 and 4, redo $\log$ records are written to an Lo log file. Requirement 4 can be implemented either by logging page modifications (i.e., modified bytes) [Li79, Cr84, MLC87, Mo89], or by writing entire page after-images as in the DB Cache method [EB84]. The first option, which is usually referred to as "entry logging", causes less log volume (i.e., saves log space) and may thus have shorter log $1 / O$ s. Note, however, that after-image logging does not cause a higher number of log $1 / O s$, given that multiple pages can be sequentially written in a single set-oriented $\mathrm{I} / 0$. On the other hand, during a warmstart, a recovery method with entry logging is slower than a method with after-image logging. This is because pages have to be fetched from the database before the update that is described in a log record can be installed, whereas after-images can be directly written into the database right after they have been read from the log. That is, after-image logging saves a substantial number of random $1 / O$ s during the warmstart. For this reason and for simplicity, we assume in the following that after-image logging is used, as it is actually implemented in DASDBS by applying the DB Cache method to page-level subtransactions. Note, however, that most considerations of this paper fit with entry logging as well.

\section{Ensuring Subtransaction Atomicity}

Requirement 3, subtransaction atomicity, is the one that makes multi-level recovery difficult. Essentially, it is handled by using page before-images as the LO undo information. Since these before-images are only needed for incomplete subtransactions, they are kept in main memory as temporary page versions in the buffer pool. This provides an efficient method for undoing a subtransaction, e.g., to resolve a page-level deadlock between subtransactions. 
Unfortunately, a complete solution for subtransaction atomicity is not quite that simple. If a dirty (i.e., modified) page were replaced in the buffer pool and written back to the database before a subtransaction completes, the before-image of the page would have to be written to disk first, according to the write-ahead logging (WAL) rule [BHG87]. This problem is circumvented in our implementation by assuming (and having implemented) a No-Steal buffer manager [HR83] that does not replace a dirty page before EOS. Note that a No-Steal policy for subtransactions is feasible since subtransactions usually have bounded length, whereas the same assumption for arbitrarily long transactions may be debatable (see [EB84] for dealing with long transactions under a No-Steal policy).

A more severe problem is that replacing a dirty page is critical even after the completion of the subtransaction that has modified the page. Again, this would violate the atomicity of a subransaction that has modified multiple pages. One solution could be to keep the before-images if a subtransaction beyond its completion. However, even keeping all before-images until EOT inot sufficient for ensuring subtransaction atomicity. To verify this claim, consider the following cenario.

iuppose that, in the example of Figure 1, the page $q$ is replaced in the buffer pool at time $t_{6}$. Vriting $q$ back to the database would violate the atomicity of subtransaction $T_{11}$, since this subtransaction has modified two pages $p$ and $q$. If a crash occured right after $t_{6}$, using the beforeimages of $T_{11}$ to reestablish the atomicity of $T_{11}$ would incidentally undo the updates of $T_{21}$ on page $p$. This would in turn violate the atomicity of $T_{21}$, and, even worse, would violate the persistence of $T_{2}$ which is already committed at time $t_{6}$.

Our solution for ensuring the atomicity of a subtransaction when a dirty page is replaced after EOS is to force the LO redo information of the subtransaction to the LO log file. The after-images of a subtransaction are written atomically, by including a special EOS flag in the header of the last page of the written after-images. This flag serves as an EOS log record.

So far, we have not discussed when the after-images of a subtransaction are written to disk. In fact, this is the most critical point of our recovery method. Because of its importance, this issue is discussed separately in Subsection 2.2.2. For now, we assume that a subtransaction's afterimages are forced to the log file immediately after EOS. While this is obviously inefficient, it is a correct multi-level recovery method and was in fact the first method that was implemented in (a former version of) DASDBS. It is worthwhile to note that this method is essentially the DB Cache method [EB84] applied to subtransactions. The DB Cache method is one of the most efficient recovery methods, and has nice properties with respect to how the log space is managed (i.e., dynamically compacted without having to take checkpoints) [EB84]. Its main drawback is that is works only in combination with page locking. This disadvantage does not hold for our multilevel transaction management, since we employ the DB Cache method only to handle subtransactions at the page level.

The log records that are written for the example of Figure 1 are shown in Figure 3. Operations with an overbar denote inverse operations. For reasons that are discussed in Subsection 2.2.3, the $L 1$ log records and the after-images that are written at $L 0$ include a subtransaction identifier in a special header field. The warmstart after a crash consists of the following two steps:

1) Redo pass:

Determine the relevant starting point in the $L 0$ redo log by looking up a special master record [EB84], and perform a forward pass on the Lo redo log. During this pass, after-images are loaded into the buffer pool and written into the database at the discretion of the buffer 


\begin{tabular}{|c|c|c|c|c|c|c|c|c|}
\hline & & 7 & 5 & & & & & \\
\hline $\begin{array}{l}\text { High-Loved } \\
\text { Undo Log }\end{array}$ & $\frac{T_{11}}{\text { Change(x) }}$ & & $\frac{T_{21}}{\text { Change }(x)}$ & $\frac{T_{12}}{\text { Change(y) }}$ & & & & $\begin{array}{c}T_{2} \\
\text { EOT } \\
\end{array}$ \\
\hline $\begin{array}{l}\text { Low-Lovel } \\
\text { Rodo Log }\end{array}$ & & $\begin{array}{c}T_{11} \\
p\end{array}$ & $\begin{array}{c}T_{11} \\
q\end{array}$ & $\begin{array}{c}T_{21} \\
p\end{array}$ & $\begin{array}{c}T_{21} \\
r\end{array}$ & $\begin{array}{c}T_{12} \\
s\end{array}$ & $\begin{array}{c}T_{12} \\
r\end{array}$ & \\
\hline
\end{tabular}

Fig.3: Log Contents for the Example of Fig.1

manager. The redo pass ensures transaction persistence and subtransaction atomicity at acceptable performance (i.e., requirements 2,3, and 4). After-images after the latest EOSflagged after-image are ignored since they belong to incomplete writes at EOS.

2) Undo pass:

After the redo pass, a backward pass is performed on the L 1 undo log. The undo pass ensures transaction atomicity. Transactions for which an EOT log record is found are winners and do thus not need any processing. For loser transactions, compensating subtransactions are performed according to the contents of their log records.

\subsubsection{Deferred Log Writes}

The multi-level recovery algorithm of the previous subsection was implemented in a former version of DASDBS [SPSW90]. This algorithm has a potential performance problem in that it may cause excessive log $\mathrm{I}$ Os for ensuring the atomicity of subtransactions. This is because after-images of a subtransaction are forced to disk immediately at EOS (which in turn requires forcing the $L 1$ undo log before, so as to observe the WAL rule). In the example of Figure 1, this means that an after-image of page $p$ is written to disk at the EOS of $T_{11}$ and the EOS of $T_{21}$, as shown in Figure 3.

While there are generic techniques to reduce these $1 / O$ costs such as batching $\log 1 / O$ s of multiple transactions [GK85, He87], there is a more fundamental way to cut down the log $1 / O$ costs of multi-level transactions. The general idea is to defer the writing of a subtransaction's afterimages until EOT rather than forcing them at EOS. This would make multi-level logging as efficient as conventional single-levellogging, e.g., the original DB Cache method [EB84]. However, deferring all LO log writes until EOT is not a correct solution. The reason is that there may be subtransactions of different transactions such that the after-image sets of the subtransactions are overlapping, i.e., have a page in common. This is possible because page locks are released at EOS. In such a situation, forcing the after-images of one subtransaction at the EOT of its parent may violate the atomicity of the other subtransaction.

Consider the example of Figure 1. Ideally, we would want to write the after-images of $T_{11}$ and $T_{12}$ not before the EOT of $T_{1}$. The EOT of $T_{2}$ requires writing the after-images of $T_{21}$, i.e., pages $p$ and $r$ as of the EOT time of $T_{2}$. Writing these pages to the $\mathrm{LO} \mathrm{log}$, however, would implicitly write the modifications that $T_{11}$ made on $p$, too. Then, if the system crashed before the EOT of $T_{1}$ (i.e., before the after-images of $T_{11}$ are written), the redo pass of the warmstart would violate the atomicity of $T_{11}$ by restoring the update on $p$ while disregarding $T_{11}$ 's update on $q$. Note that this problem would arise also with entry logging rather than after-image logging because subtransactions of different transactions may have modified a common byte through commutative high-level update operations.

The same problem, in a slightly different flavor, arises with respect to subtransaction $\mathrm{T}_{12}$. For efficient memory use, the after-images reside in regular buffer frames rather than being copied 
to a separate $L 0$ log buffer. For the same reason, we do not want to keep multiple versions of a page in the buffer pool when there is no active subtransaction that has modified the page; that is, the temporary before-images are discarded at EOS. Therefore, at the EOT of $T_{2}$, only one version of page $r$ resides in the buffer pool. This version contains the updates of the completed subtransaction $T_{12}$ even though $T_{12}$ was serialized after $T_{21}$. Thus, writing this after-image of $r$ to the $\mathrm{LO} \log$ file would violate the atomicity of $\mathrm{T}_{12}$.

These and other related problems have been discussed more rigorously in [WHBM90] and have lead to a solution that is based on the notion of persistence spheres. The persistence sphere PS $\left(T_{1 j}\right)$ of a subtransaction $T_{i j}$ is defined as follows:

- PS $\left(T_{1 \mid}\right)$ contains all pages that were modified by $T_{i j}$ and have not yet been written to the $L 0$ log file since their last modification by $\mathrm{T}_{\mathrm{ij}}$.

- If there is a page $p$ in PS(Til) and a subtransaction $T_{k l}$ such that $p$ is also in $P S\left(T_{k l}\right)$ or $T_{k l}$ has read $p$ after the EOS of $T_{i j}$, then PS $\left(T_{i j}\right)$ contains all pages of $P S\left(T_{k l}\right)$; that is, the persistence spheres of the two subtransactions are merged.

The persistence sphere.PS $\left(T_{i}\right)$ of a transaction $T_{1}$ is the union of the persistence spheres of its subtransactions. Now, our solution to the deferred log write problem is the following. At the EOT of a transaction $T_{1}$, all pages in the persistence sphere of $T_{1}$ must be written to the Lo log. In addition, replacing a dirty page $p$ in the buffer pool requires forcing to the log all pages in the persistence sphere of the last completed subtransaction that modified $p$. In the example of Figure 1, the subtransaction $T_{21}$ is mutually dependent on $T_{11}$ as well as $T_{12}$. Thus, at the EOT of $T_{2}$, the persistence sphere of $T_{2}$ contains the pages $p$ and $r$ that were modified by $T_{2}$ 's own subtransaction $T_{21}$ and the pages $q$ and $s$ that were modified by $T_{11}$ and $T_{12}$, respectively.

Persistence spheres are written atomically to the LO log file, by setting the EOS flag in the header of the last page (see Subsection 2.2.1). A persistence sphere may contain updates of completed subtransactions that belong to incomplete transactions. These subtransactions will have to be compensated if the system crashes before the EOT of their parent. To be able to do so, the L1 undo log must be forced before the LO $\log 1 / O$ (thus observing the WAL rule). In addiion, it seems that immediately after the writing of the persistence sphere is completed, another $L 1 \log \mathrm{V} / \mathrm{O}$ is often necessary in order to force the EOT log record of the committing transaction that caused the writing of the persistence sphere. Fortunately, this second L1. log $1 / O$ can be avoided by including an additional EOT flag and the number of the committing transaction in the header of the last page of the persistence sphere. An EOT log record is nevertheless created in the L1 log buffer pool, but need not be forced before the next compaction of the LO log file that would discard the after-image that contains the EOT flag. On the other hand, it may turn out, at the EOT of a transaction, that all after-images of that transaction have already been written to the LO log as parts of the persistence spheres of other transactions. In this case, the EOT log record of the committing transaction is forced to the $L 1$ log disk, rather than performing an additional $L O \mathrm{log}$ write. The log records for the example of Figure 1 are shown in Figure 4.

\section{Managing Persistence Spheres}

In DASDBS, persistence spheres are implemented by means of the following types of control blocks:

- For each active transaction, a transaction control block (TCB) contains pointers to the subtransaction control blocks of its own subtransactions.

- For each subtransaction of an active transaction, a subtransaction control block (STCB) contains writeset pointers to the buffer frame control blocks of the pages that were modified. 


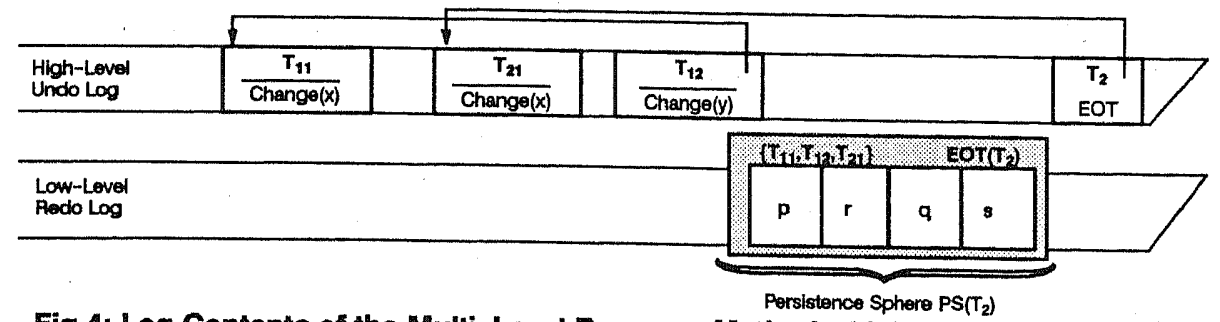

\section{Fig.4: Log Contents of the Multi-Level Recovery Method with Deferred Log Writes}

by the subtransaction, and readset pointers to the buffer frame control blocks of the pages that were only read. The writeset pointers have backward pointers associated with them; that is, the frame control block of a page points to the STCBs of all subtransactions that modified the page. An STCB is discarded as soon as the subtransaction's after-images are written to the $L O$ redo log file.

- For each buffer frame, a frame control block (FCB) contains status information about the page that is held in the buffer frame. The status can be

- "modified", which means that an incomplete (i.e., running) subtransaction has modified the page,

- "dirty", which means that a completed subtransaction has modified the page but the modified page has not yet been written to the LO log nor to the database,

- "forced", which means that a completed subtransaction has modified the page and the modified page has already been written to the LO log, or

- "clean", which means that no incomplete transaction has modified the page and an identical version of the page resides in the database.

FCBs with status "modified" contain a bfim pointer to another FCB that points to a beforeimage frame. For frequently modified pages, the before-image FCB is usually a "dirty" FCB of a previously completed subtransaction.

- Finally, for each persistence sphere, a persistence sphere control block (PSCB) points to the STCBs of those subtransactions that constitute the persistence sphere. These pointers have backward pointers associated with them, i.e., an STCB also points to its PSCB. Note that each STCB belongs to exactly one PSCB. A PSCB and the STCBs that it points to are discarded as soon as the persistence sphere has been written to the log.

Managing persistence spheres by means of these control blocks is illustrated in Figure 5 , which is based on the example of Figure 1. Figure 5 shows snapshots of the necessary control blocks at different points of time. Figure 6 shows pseudocode for the complete handling of BOT, BOS, page modifications (i.e., fixing a page for write), EOS, EOT, and dirty page buffer replacements. Note that these procedures ensure that, at each point of time, a "dirty" page belongs to exactly one PSCB. That is, if the page had been modified by multiple completed subtransactions, then the persistence spheres of these subtransactions would have been merged already by the corresponding EOS procedure calls. Further note that the persistence spheres of a transaction's subtransactions are not merged before EOT or the occurence of a page dependency (as checked at EOS). This "just-in-time" merging of persistence spheres minimizes the impact of transitive dependencies between subtransactions. This in turn keeps the number of pages in a persistence sphere as small as possible, and thus avoids excessively long log writes that might adversely affect transaction response time. 


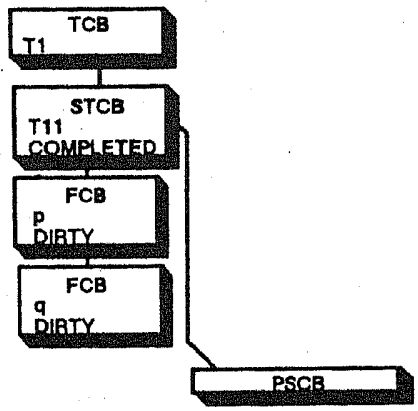

a) at time $t_{1}$ (i.e., after $\operatorname{EOS}\left(T_{11}\right)$ )

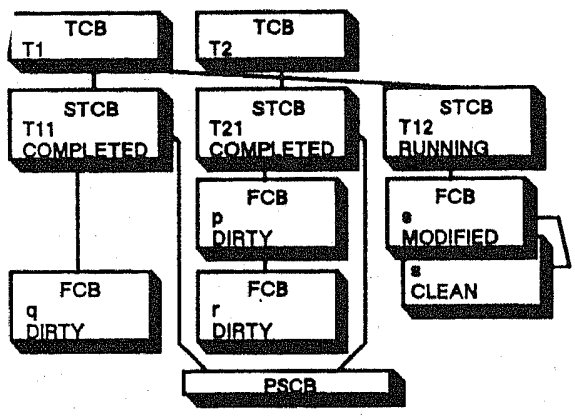

c) at time $t_{3}$ (i.e., after $\operatorname{EOS}\left(T_{21}\right)$ )

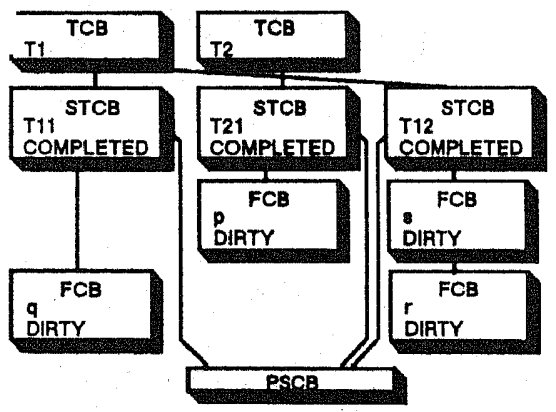

e) at time $t_{5}$ (i.e., after $\operatorname{EOS}\left(T_{12}\right)$ )

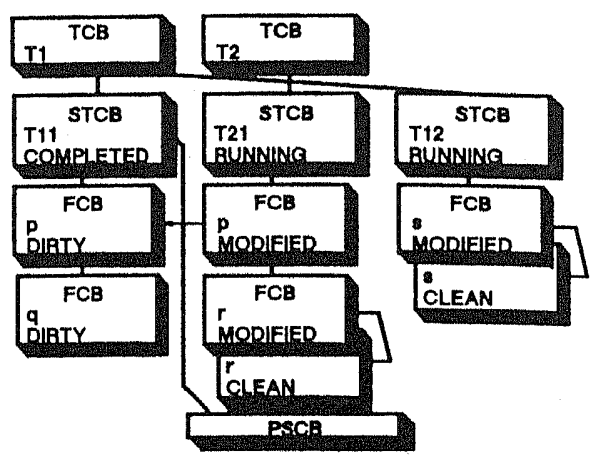

b) at time $t_{2}$ (i.e., right before EOS $\left(T_{21}\right)$ )

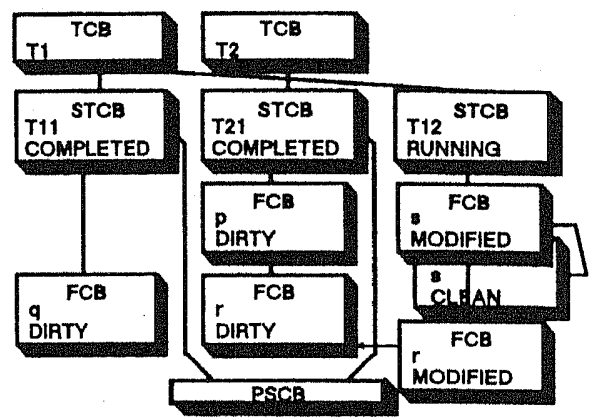

d) at time $t_{4}$ (i.e., right before $\operatorname{EOS}\left(T_{12}\right)$ )
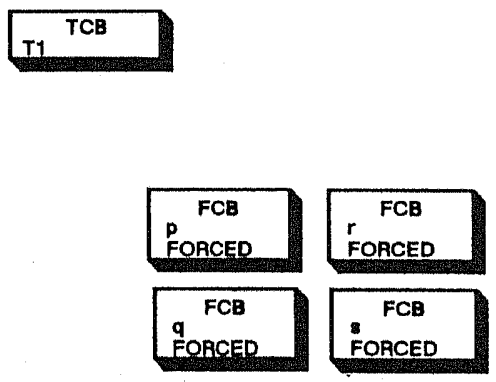

f) at time to (l.e., after EOT(T2))

Fig.5: Snapshots of Control Blocks for the Scenario of Fig.1 
BOT(T):

Create TCB

$\operatorname{BOS}\left(\mathrm{T}_{\mathrm{j} j}\right)$ :

Create STCB

Attach STCB $\left(T_{i j}\right)$ to $\operatorname{TCB}\left(T_{i}\right)$

Fix_for_Write $\left(T_{1,}, q\right)$ :

Allocate a new buffer frame with FCB $f$

Copy $q$ into the new frame

Attach the original FCB of $q$ to $f$

Set the status of $f$ to "modified"

Attach $f$ to $\operatorname{STCB}\left(\mathrm{T}_{\mathrm{j}}\right)$. writeset

$\operatorname{EOS}\left(T_{1 j}\right):$

for each

do

$$
\text { "modified" FCB } f \text { in STCB }\left(T_{i j}\right) \text {. writeset }
$$
or "dirty" FCB $f$ in STCB $\left(T_{i j}\right)$.readset

for each STCB $s$ that points to $f$

de

if $s$ points to a $P S C B$ then

ed fi

Add this PSCB to a list L of PSCBs

if status of $f$ is "modified" then

Disconnect $f$ from all STCBs except

fi the last writer STCB $\left(T_{i j}\right)$

ed

Set the status of FCB $f$ to "dirty"

Drop the FCB bfim(f)

if $L$ is not empty then

Merge all PSCBs in L by attaching all

STCBs of the 2nd through last PSCB in L else to the first PSCB $p$ in $L$

fi

Create a new PSCB p

Attach STCB $\left(T_{i j}\right)$ to PSCB p

\section{Replace_Dirty_Page(q):} for each STCB s that points to $\mathrm{FCB}(q)$ de

if $s$ points to a PSCB $p$ then Write persistence sphere $p$ Write page $q$ back into the database fi Set the status of FCB(q) to "clean" od

$\operatorname{EOT}\left(\mathrm{T}_{1}\right)$ :

for each STCB s that points to TCB $\left(T_{i}\right)$ do

if $s$ points to a PSCB $p$ then

fi Add this PSCB to a list L of PSCBs

if $L$ is not empty then

Merge all PSCBs in L by attaching all STCBs of the 2nd through last PSCB in L to the first PSCB p 1 in L fi Write persistence sphere $\mathrm{p} 1$ od

Drop TCB $\left(T_{i}\right)$

Write_Persistence_Sphere(p):

Collect a list $L$ of "dirty" FCBs by traversing all FCBs of all STCBs that are attached to PSCB p

Write the pages that are pointed to by the FCBs in L to the LO log, and set the status of the FCBs to "forced" Drop all STCBs that point to $p$ Drop PSCB $p$

Fig.6: Pseudocode for Multi-Level Logging

\subsubsection{Warmstart Procedure}

A nice property of our multi-level recovery algorithm is that, even with deferred log writes, the warmstart procedure after a crash is fairly simple. In fact, the warmstart processing can be directly adopted from the multi-level recovery algorithm without deferred log writes, as sketched in Subsection 2.2.1. During the redo pass, the after-images of the LO redo log are loaded into the buffer pool and are written into the database according to the buffer manager's write policy. Thus, all completed transactions and all subtransactions that were in the persistence sphere of 
a completed transaction are redone. During the subsequent backward pass on the L1 undo log, compensating subtransactions are invoked for those subtransactions that belong to loser transactions.

During the undo pass, a problem arises because the high-level undo log record of a subtransaction is always forced to the $L 1$ log file before the subtransaction's after-images are written to the LO log file. Because these two write operations are not performed together as a single atomic event, the undo pass during a warmstart may encounter an undo log record of a subtransaction the after-images of which were not yet written to the LO log file when the crash hit. For example, in the scenario of Figure 4, suppose that the system crashes right after the undo log record for $T_{12}$ was written to the $L 1 \log$ file. Then, since $T_{11}, T_{21}$, and $T_{12}$ will not be redone during the warmstart, we must take care that the inverse high-level operation for these subtransactions will have no effect on the database. To guarantee this property even for arbitrary high-level operations, the recovery manager itself must figure out which high-level log records must be skipped during the undo pass. Note that this problem is essentially the problem of ensuring idempotence for non-idempotent operations such as "increment" operations. If one views the fact that a subtransaction's updates were lost in a crash as a fictitious undo operation, then we must guarantee that a "second". execution of the undo operation is prohibited or has no effect.

Our solution to the described problem is to store the numbers of the executed subtransactions in both the LO redo log records and the L1 undo log records, as shown in Figures 3 and 4 . These subtransaction numbers can be viewed as log sequence numbers (LSNs) of the L1 log. If the Lo log were based on entry logging with entries of the type "shift 100 bytes by 10 bytes to the right" describing non-idempotent page-level operations, then an additional handshake would be needed between the $L 0 \mathrm{log}$ and the database itself so as to provide idempotent redo. This additional handshake is usually implemented by storing the highest $L S N$ of a page's $L 0$ update log records in the header of the page [Gr78, Cr84, Mo89]. By comparing the LSN of a log record with the LSN of the page, the warmstart procedure can decide whether the log record must be skipped or not.

Implementing the handshake between the LO log and the L1 log is a little bit more difficult. The extra complexity comes from the fact that the order of the subtransactions' after-images in the LO redo log may be different from the order of the same subtransactions' log records in the L1 undo log. Thus, during the redo pass of the warmstart, it is not sufficient to keep track of the highest subtransaction number (i.e., L1 LSN) that is contained in the L0 log. Rather we must collect a list of "winner subtransactions" that is afterwards used by the undo pass for checking the applicability of the L1 log records.

In the multi-level recovery method without deferred log writes, each after-image in the LO log belongs to exactly one subtransaction. With deferred log writes, each after-image belongs to one persistence sphere which may consist of multiple subtransactions. Hence, we actually record a list of subtransaction numbers that is spread across the headers of a persistence sphere's after-images rather than merely a single subtransaction number. If this list becomes unusually long, an additional page with such bookkeeping information is included in the setoriented $\mathrm{V} / \mathrm{O}$ that writes the persistence sphere. The header of the last after-image of a persistence sphere contains the number of the committing transaction that caused the writing of the persistence sphere. The committed transaction numbers are also collected during the redo pass, and are needed by the undo pass to handle the case of non-forced (and thus missing) EOT log records in the L1 log (see Section 2.2.2). 


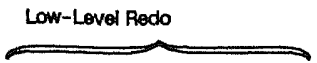

$W(r) \quad W(q) \quad W(s)$
High-Level Undo for T1

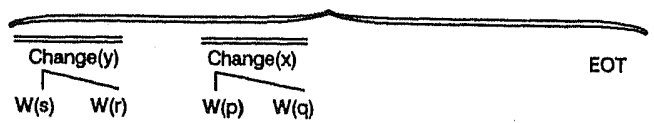

Fig.7: Operations During the Warmstart

$\begin{aligned} & \text { Transaction } \\ & \text { Backward Chain }\end{aligned}$
$\begin{aligned} & \text { High-Lovel } \\ & \text { Undo Log }\end{aligned}$

Fig.8: Log Records Written During the Warmstart

\section{Logging During the Warmstart}

During the redo phase of a warmstart, no logging is necessary since restoring page afterimages is idempotent and can therefore be repeated as often as necessary. During the undo phase, however, LO redo logging must again be in effect to ensure the atomicity of the executed compensating subtransactions. In addition, to keep track of the progress during the undo phase and to be able to handle repeated warmstarts, L1 undo log records are written for the executed compensating subtransactions. For example, Figures 7 and 8 show the operations that are executed and the log records that are written during the warmstart for the scenario of Figures 1 and 4. Operations with a double overbar denote the inverses of inverse operations.

In this approach, compensating subtransactions and regular subtransactions are treated uniformly for simplicity. Thus, undoing a transaction is actually not distinguishable from performing forward recovery. Therefore, at the end of undoing a transaction, an EOT log record is written as though the transaction were normally completed. If, in the example of Figure 8 , the system crashed once more right before the EOT $\left(T_{1}\right)$ log record is written, then the following warmstart would redo the subtransactions $T_{11}$ through $T_{14}$. Then, if the after-images of $T_{13}$ and $T_{14}$ did not include the EOT flag (e.g., because the rollback was not yet complete), our recovery manager would undo both the compensating subtransactions $T_{14}$ and $T_{13}$ and the regular subtransactions $T_{12}$ and $T_{11}$ by following the "transaction backward chain" of $L 1$ log records.

An optimization of the described implementation would be to apply the technique of [Mo89] that avoids undoing an undo operation (i.e., compensating a compensating subtransaction) by following an additional "compensation backward chain" between the L 1 log records of a transaction. This technique guarantees that repeated crashes do not cause increasingly longer warmstarts, and allows resolving top-level deadlocks by partially rolling back a transaction. ${ }^{*}$ It seems that incorporating these features in our implementation would be fairly straightforward

\footnotetext{
\#ote that page-level deadlocks can be handled more easily by rolling back and restarting one or more subtransactions, l.e., by exploiting the nested transaction structure.
} 
by simply adding the compensation backward chain, as shown in Figure 8 . In the example, the L1 log record of $T_{13}$ would point to $T_{11}$, i.e., the predecessor of the subtransaction that is compensated by $T_{13}$; and $T_{14}$ would have a nil pointer because it compensates $T_{11}$ and $T_{11}$ is the transaction's first subtransaction. The processing of the L1 undo log would follow this additional compensation backward chain until a log record is encountered that corresponds to a subtransaction that is not among the "winner subtransactions" of the redo phase. As this log record is skipped (see above), we also ignore its compensation backward pointer and rather follow the regular transaction backward chain.

\section{Adding Intra-Transactlon Parallelism}

Advanced DBMS applications such as engineering or document management have a high potential for parallelism within a single transaction [DG90, Du87, HSS89, HPS90]. Such intra"ansaction parallelism is a key technology for speeding up both retrieval and set-oriented uplate operations on complex objects. Similarly, applications that update large amounts of lerived data and/or check complex integrity constraints can substantially benefit, too [HK89] see Section 1 for an example). We have extended our implementation of multi-level transacion management so that it can also deal with parallel subtransactions of a single transaction. Implementing these extensions has been fairly straightforward. Multi-level transaction management, by its modular nature, deals uniformly with subtransactions at the page level, regardless of whether two subtransactions belong to different transactions or to the same transaction. Thus, adding intra-transaction parallelism required only one additional component for scheduling the subtransactions within a transaction, and it required changes to the process architecture of DASDBS. In the following two subsections, these modifications are briefly discussed.

\subsection{Scheduling of Subtransactions}

The newly implemented scheduling component expects that the programmer of a transaction program specifies the precedence orders between the subtransactions of a transaction. Generally, two subtransactions have no precedence order if there is neither a control flow nor a data flow dependency between them and if they do not potentially conflict at the object level. However, subtransactions that are ${ }^{\text {independent }}{ }^{n}$ in the above sense are allowed to have potential conflicts at the page level. It is still reasonable to execute such subtransactions in parallel, because a potential conflict does not necessarily mean that a lock conflict will actually occur. Even if there is a page-level lock conflict between two parallel subtransactions of the same transaction, it may still be beneficial, in terms of response time, to exploit the possible parallelism to the largest possible extent rather than serializing the subtransactions in advance. In the worst case, a page-level deadlock can involve two or more subtransactions of the same transaction. This case is recognized by the lock manager and handled in the same way as a page-level deadlock between subtransactions that belong to different transactions. That is, one or more subtransactions are rolled back and (automatically) restarted; it is not necessary to abort the entire transaction. * Of course, if the deadlock could be foreseen before the subtransactions start executing (i.e., if the probability of a deadlock is estimated to be high), the critical subtransactions should better be serialized in advance.

From the specification of the precedence orders between subtransactions, a Petri-net-like precedence graph is constructed. This graph is used for driving the parallel execution of the subtransactions. That is, a subtransaction is invoked by the scheduler when all its predecessors in the precedence graph are successfully completed. It is planned to enhance the sched-

\footnotetext{
* This advantage would, of course, hold for any other nested transaction model as well (e.g., [Mo85, HPS90]).
} 
uler so that it takes into account estimates about the resource consumption and the locking behavior of a subtransaction. The goal is to schedule eligible subtransactions in such a way that the utilization of processors and the utilization of disks are approximately balanced (cf. [Pi90, MS91]). Furthermore, the scheduling of subtransactions should avoid data-contention bottlenecks and especially deadlocks that can be predicted in advance.

\subsection{Process Architecture}

DASDBS has a process-per-transaction architecture; that is, each transaction is executed in a separate process, with newly arriving transactions reusing existing processes. All global data structures (i.e., buffer frames, control blocks for buffer management, locking, logging, etc.) are allocated in shared memory. In the original implementation, each process had only one thread of control for sequentially executing all subtransactions of a transaction. This has been extended by spawning a light-weight process for each subtransaction that is to be executed. These light-weight processes are provided by the $\mu$ System parallel programming library [BS90] that we used in the implementation. Light-weight processes are called $\mu$ tasks in the $\mu$ System; we will refer to them simply as "tasks". Such tasks are executed within a "cluster" of one ore more heavy-weight (i.e., Unix) processes. The processes within a cluster are called "virtual processors" in the $\mu$ System. All processes of all clusters share the same heap, for which the $\mu$ System provides the memory management.

As we have to deal with both inter- and intra-transaction parallelism, we generate a cluster of processes for each concurrently executing transaction, in accordance with the original process-per-transaction architecture. The new process architecture is illustrated in Figure 9 . The number of processes in a cluster is dynamically adjusted so that it is always equal to the number of simultaneously active tasks, that is, parallel subtransactions within a transaction. Because of the high costs of process creation and destruction associated with this dynamic mechanism, we also support an alternative in which the number of processes in a cluster is set to the maximum number of tasks that can be simultaneously active. This number of processes is set already at the beginning of a transaction, and all processes are kept until the transaction completes.

A major point of the described process architecture is that it also allows using less processes in a cluster than there are concurrently executing tasks. This option, which is provided by the $\mu$ System for each cluster individually, is useful if some of the tasks are $1 / 0$-intensive so that the number of tasks that require processors is less than the number of executing tasks. ${ }^{*}$ In addition, the combination of inter- and intra-transaction parallelism may require limiting the total number of processes in the process clusters of the concurrently executing transactions. This sort of load control or throttling is essential for avoiding excessive context-switching (of heavyweight processes) as well as other thrashing-like situations like excessive memory contention and data contention. The goal that we are pursuing in the long run is to adjust the number of processes in the clusters of the transactions to the current load situation dynamically and automatically.

\footnotetext{
The $\mu$ System uses additional process clusters to provide asynchronous $1 / O$ [BS90].
} 


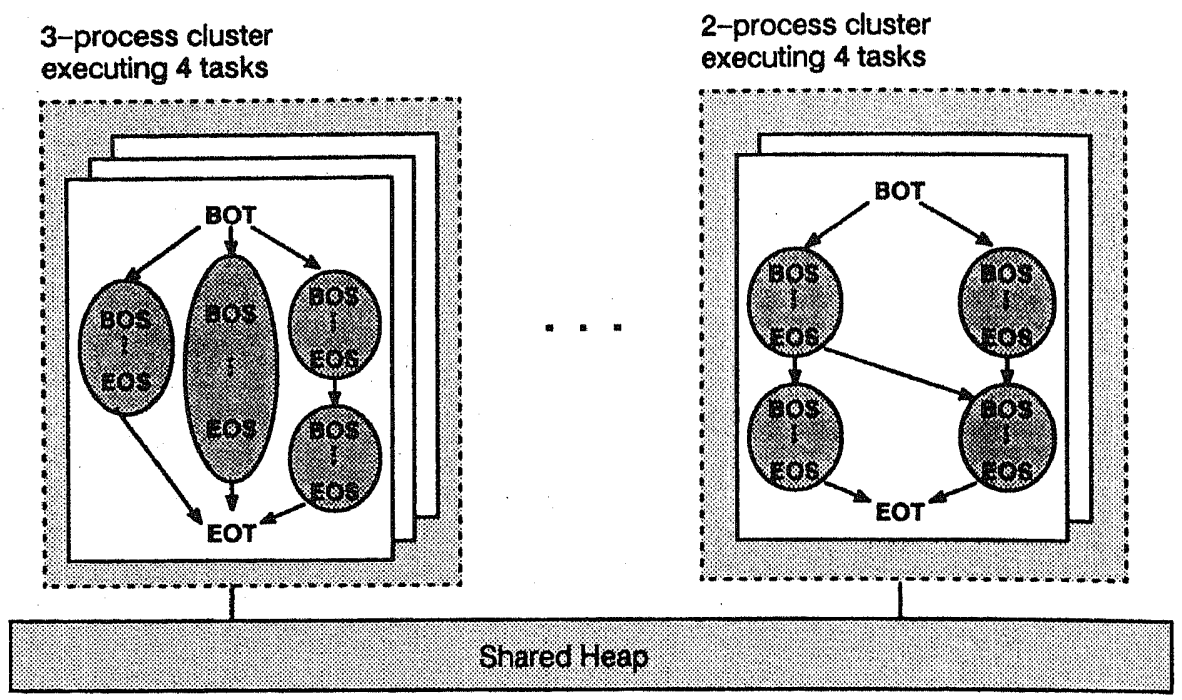

Fig.9: New Process Architecture of DASDBS

\section{Performance Evaluation}

\subsection{Description of the Experiments}

In this subsection, we describe the experiments that were performed to evaluate the performance of our algorithms for multi-level transaction management. We compared the following three strategies, all of which are implemented in DASDBS:

- strategy S1,

page-oriented single-level transaction management, using strict two-phase locking on pages and the DB Cache method for recovery,

- strategy S2, two-level transaction management with log writes at each EOS, and

- strategy S2/PS,

two-level transaction management with deferred log writes based on the notion of persistence spheres, as described in Section 2.

Since the logging overhead was one of the main aspects that we wanted to investigate, we summarize the principal log $1 / 0$ costs of the above three strategies in Figure 10.

Our performance evaluation is based on a synthetic benchmark which follows some ideas proposed in the complex-object benchmarks of [An90] and [DFMV90]. The benchmark has the following characteristics, as illustrated in Figure 11.

- Our test database consists of 1000 complex objects (COs) each of which consists of 1000 "own" subobjects (SOs) and 100 references to "foreign" subobjects, l.e., subobjects that are owned by other complex objects. Thus, SOs can be referentially shared by multiple $\mathrm{COs}$; however, each $\mathrm{SO}$ is owned by exactly one $\mathrm{CO}$. The foreign SO references of a $\mathrm{CO}$ are generated by selecting a $\mathrm{CO}$ according to an $80-20$ rule and a SO within the selected 


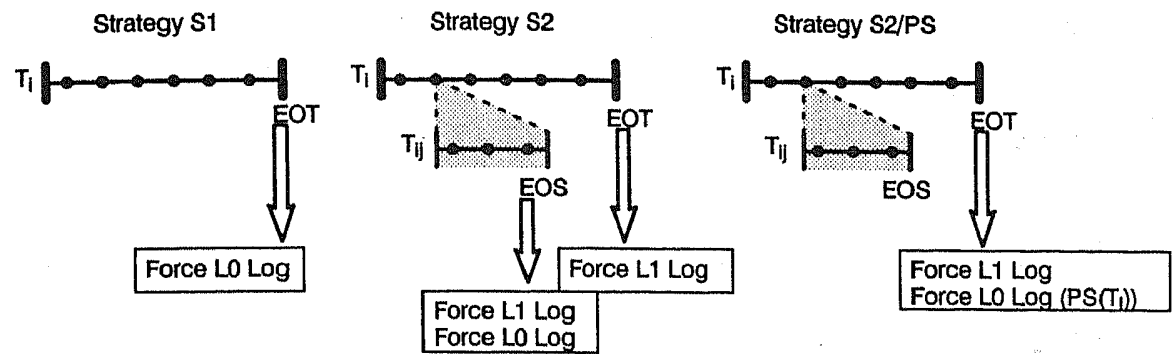

Fig.10: Log 1/O Costs of Different Recovery Strategies

CO according to a $50-50$ rule. That is, $80 \%$ of the foreign SO references point to SOs that are owned by $20 \%$ of the COs in the database. This reflects the skewed distribution of object relationships in most real-life applications. In our benchmark, the 80-20 rule and the 50-50 rule were implemented by applying a linear transformation to a normal distribution of random numbers.

The 1000 "own" SOs of a CO constitute a storage cluster that consists of 10 contiguous pages, with a page size of $2 \mathrm{KBytes}$. The first page of each storage cluster contains the $\mathrm{CO}$ header, i.e., a directory of SO references. The total database size is 10000 pages, i.e., 20 MBytes.

- The workload of our benchmark consists of a single transaction type which performs $c$ complex high-level operations each on a different $\mathrm{CO}$. Each of these synthetic high-level operations accesses $O$ own subobjects and $f$ foreign subobjects of a CO. A subobject is modified with probability $u$. These updates do not affect the $C O$ header; that is, the header page of a $\mathrm{CO}$ is read-only to avoid an obvious data-contention bottleneck in the benchmark. The COs that are processed by a transaction are selected according to an 80-20 rule, the own SOs within a $\mathrm{CO}$ are selected according to a 50-50 rule, and the foreign SOs are selected to a uniform distribution as the references themselves are already non-uniformly distributed (see above). According to [Hä87], this skewed distribution is rather conservative compared to the access skew of many real-life applications.

In the multi-level transaction management strategies S2 and S2/PS, each high-level operation on a $\mathrm{CO}$ corresponds to a subtransaction. At the object level, each high-level operation acquires shared locks on the set of accessed SOs, using object identifiers as the actual lock items. For modified SOs, these locks are acquired in exclusive mode. At the page level, all accessed pages are locked in shared mode, with conversions to exclusive locks for modified pages. In the strategies S2 and S2/PS, all page locks are released at EOS (i.e., when a high-level operation completes), whereas in the single-level transaction management strategy $\mathrm{S} 1$, all page locks are held until EOT.

The experiments were designed as a stress test for transaction management on complex objects, with a small database and fairly long update transactions. All measurements were performed with DASDBS running on a 12-processor Sequent Symmetry shared-memory computer, with a page buffer pool of $2 \mathrm{MBytes}$. Each run of the experiments was driven by a fixed number of processes that execute transactions. This number of processes restricts the maximum number of transactions that can be concurrently executing, and is referred to as the degree of multiprogramming (DMP). In the experiments, the DMP was systematically varied for different runs. 
1000 complex objects (COs)

each with 1000 "own" subobjects (SOs)

and references to 100 "foreign" SOs

(10 pages per $\mathrm{CO}$ )

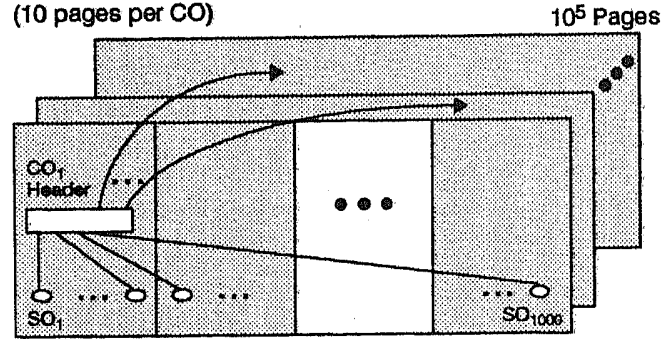

Page 1 Page 2

Page 10 c operations on COs

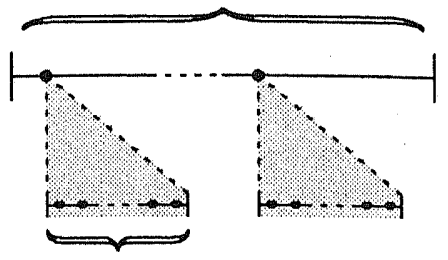

0 accesses to "own" SOs and $f$ accesses to "foreign" SOs both with update probability $u$

Fig. 11: Database and Workload of the Performance Experiments

\subsection{Performance Results for Disjoint Complex Objects}

In this section, we discuss the performance results for the case without accesses to foreign subobjects (i.e., $f$ was set to 0 ). We first discuss the results of a "baseline experiment" with $c=12$ complex-object operations per transaction, $0=10$ own-subobject accesses per complex-object operation, and update probability $u=20 \%$. We have also performed a sensitivity analysis of these parameters, as discussed below. In the following, we discuss the key observations from these experiments.

- Overall performance:

In all experiments, both two-level strategies S2 and S2/PS clearly outperformed the one-level strategy S1. Transaction throughput and response time were improved by factors of up to 2.5 (i.e., more than two times higher throughput) and 2.4 (i.e., more than two times shorter response time). Figures $12 \mathrm{a}$ and $12 \mathrm{~b}$ show throughput and response time as a function of the DMP, where the DMP was varied between 1 and 20. Maximum throughput was reached at a DMP of 12. Detailed figures for this case are given in Figure $12 f$.

- Lock conflicts:

The performance gains of the two-level strategies result from the fact that the performance of $\mathrm{S} 1$ is limited by data contention whereas S2 and S2/PS have relatively few lock conflicts (as shown in Figure 12f for DMP 12). The observed conflict rate of 1.6 percent for strategy S1 at DMP 12 may appear acceptably low. However, the specific page reference pattern of our benchmark, with high locality within a complex object, seems to underrate the impact of the lock conflict probability. In fact, the total time that a transaction, on average, spent waiting for a lock is a more significant metric in this experiment. For example, with strategy S1 and a DMP of 12, an average transaction spent about 36 seconds waiting for locks, which is about 60 percent of a transaction's response time. With strategies S2 and S2/PS, on the other hand, this lock wait time was reduced to less than 3 seconds per transaction. Figure $12 \mathrm{c}$ shows the total lock wait time of all three strategies as a function of the DMP.

- Log 1/Os:

As the simple two-level strategy $\mathrm{S} 2$ performed log $1 / O$ s for each update subtransaction, its $\log 1 / O$ rate was dramatically higher than that of strategy $\$ 1$ (see Figure 12d). This disad- 
vantage of $\mathrm{S} 2$ was almost completely eliminated by strategy S2/PS. For example, at a DMP of 12, strategy S2/PS had about 2.7 times more page-level log I/Os than strategy S1; however, as it achieved 2.5 times the throughput of $S 1$, the $\log 1 / O$ rates of the single-level strategy and the improved two-level strategy are actually quite comparable. Note that these results reflect the relative $1 / O$ performance of the investigated strategies. As for absolute performance, the log $\mathrm{I} / \mathrm{O}$ rate did not have a significant effect on throughput or response time in any of our experiments. Even with strategy S2, the excessive number of $\log 1 / O$ s caused only about $5 \%$ utilization of each of the LO log disk and the L1 log disk. Keep in mind, however, that with more or faster CPUs, log $\mathrm{V} / \mathrm{O}$ could eventually become a performance-limiting factor. Then the savings in log 1/Os that strategy S2/PS achieved would become a crucial performance advantage.

Strategy S2/PS was even superior to strategy $\mathrm{S} 1$ in terms of the number of pages that are written in one page-level $\log 1 / O$. Because update subtransactions are dynamically combined into persistence spheres, it was often the case that a page that was modified by multiple subtransactions of different transactions was written to the log only once. This main feature of our improved multi-level logging approach led to an effect similar to group commit. With strategy S2/PS, on average only 19.9 pages rather than 22.3 pages were written in one $\mathrm{LO} \log \mathrm{I} / \mathrm{O}$, at a DMP of 12. As the decreasing average persistence sphere size in Figure 12e shows, this nice effect increases with the DMP. Note, however, that, in contrast to group commit, our method does not impose any delays on transaction commits other than the log I/O itself. In fact, group commit and our deferred log write approach are orthogonal steps toward reducing log $1 / O$ costs.

- Penformance impact of internal latches:

As the throughput and response time curves in Figures 12a and 12b show, strategy S2/PS performs slightly better than strategy S2. Even though one might think that this is the effect of the savings in log $1 / O s$, the absolute costs of $\log 1 / 0$ are actually negligible in both strategies. Rather the performance difference is because strategy S2/PS saves calls to the buffer manager as it defers the writing of after-images. This reduces some CPU overhead, and decreases the contention on internal latches that are used to synchronize the access to the buffer manager's frame control blocks (see also [GT90a] for similar experiences). Such latch contention is also the major reason for the drop of performance that both $\mathrm{S} 2$ and S2/PS suffer when the DMP exceeds 12 (i.e., the number of processors). Since we implemented latches by spin locks [GT90b], latch contention actually led to wasted CPU cycles; and since the CPU utilization was almost $100 \%$ at DMP 12, increasing the DMP beyond 12 caused a significant decrease of performance.

- Sensitivity of baseline parameters:

We performed additional experiments to study the sensitivity of the various parameters of our baseline experiment. In particular, we varied the update probability $u$, the number $o$ of own-subobject accesses per complex-object operation, and the number $c$ of complexobject operations per transaction. The results are shown in Figure 13. These experiments essentially reconfirmed the observations discussed above. In interpreting the slope of the curves, one should note that the number of modified pages per complex-object operation increases only slowly with the number of updated subobjects because of the high locality within a complex object. 


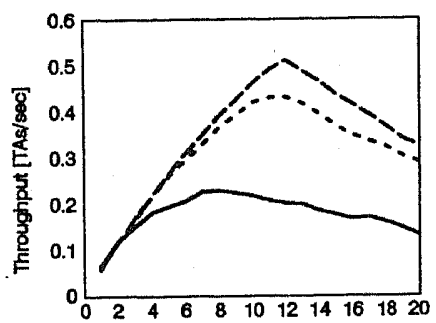

a) Throughput

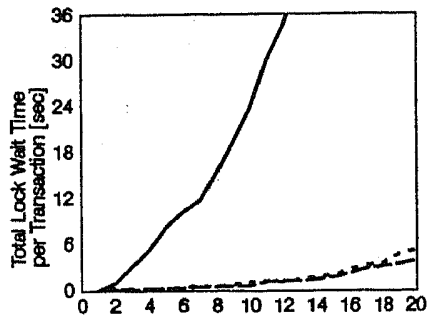

c) Total Lock Walt Tme

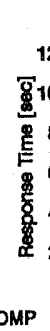

DMP

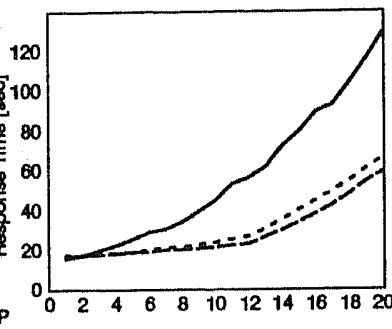

b) Response TIme

DMP

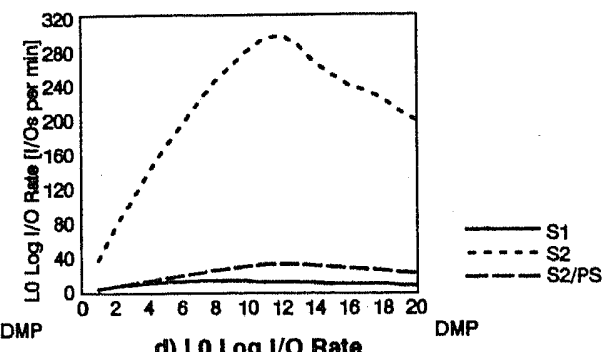

d) LO Log I/O Rate

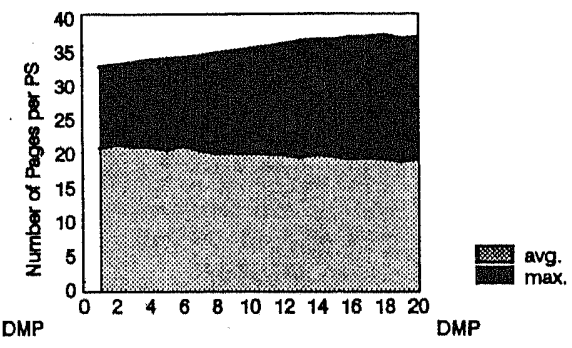

e) Persistence Sphere Slze

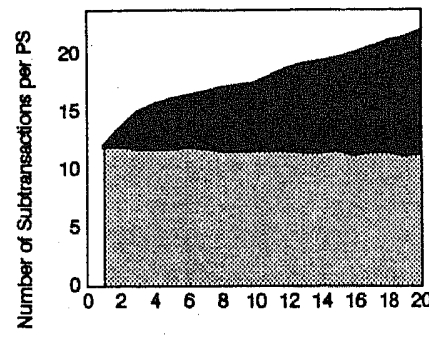



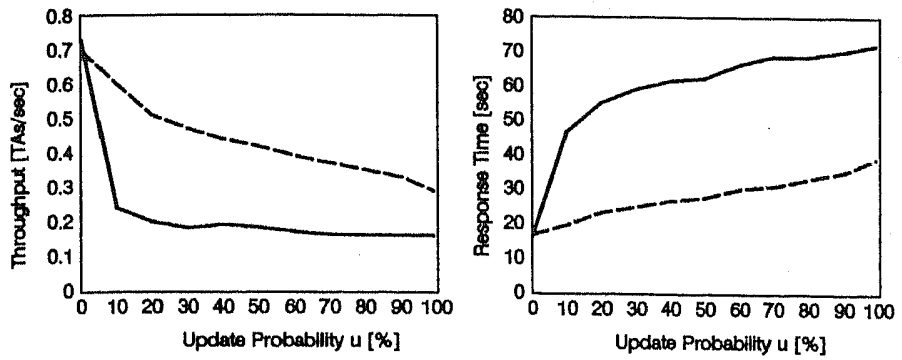
$c=12$ (number of
$=$ CO operations)
own SO accesses)
$f=0$ (number of
$D M P=12$ (degree of
toreign SO accesses)
multiprogramming

a) Throughput and Response Time with Varying Update Probabillty (u)
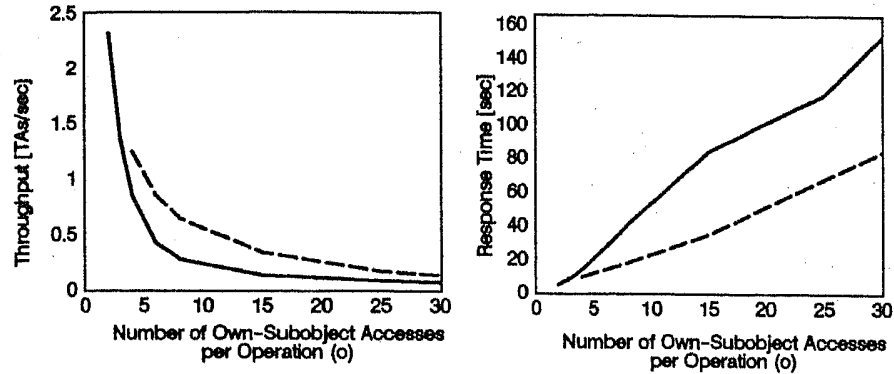

$$
\begin{array}{cl}
c=12 & \text { (number of } \\
f=0 & \begin{array}{l}
\text { CO operations) } \\
\text { (number of } \\
\text { forelgn SO accesse }
\end{array} \\
u=20 \% & \text { (update } \\
\text { probability) } \\
\text { DMP }=12 \begin{array}{l}
\text { (degree of } \\
\text { multiprogramming) }
\end{array}
\end{array}
$$

b) Throughput and Response Tlme with Varying Number of Own-Subobject Accesses (0)
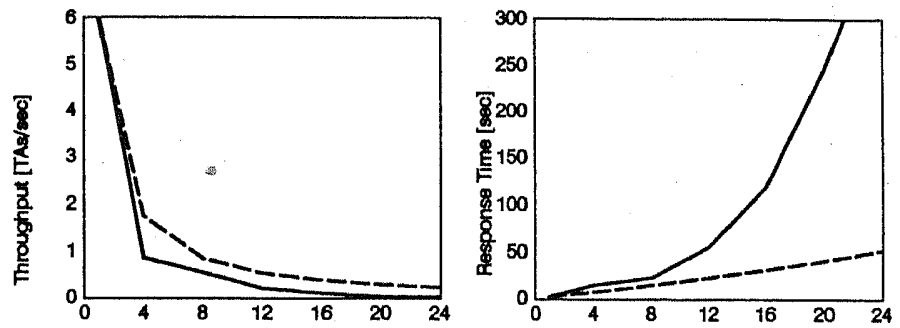

$$
\begin{array}{cl}
\hline=10 & \text { (number of } \\
& \text { own SO accesses) } \\
& \text { (number of } \\
& \text { foreign SO accesses) } \\
u=20 \% & \text { (update } \\
\text { probability) } \\
\text { DMP }=12 \begin{array}{l}
\text { (degree of } \\
\text { multiprogramming) }
\end{array}
\end{array}
$$

Number of Complex-Object Operations (c)

Number of Complex-Object Operations (c)

c) Throughput and Response Time with Varying Number of Complex-Object Operatlons (c)

Fig. 13: Sensitivity of Baseline Parameters with Disjoint Complex Objects 


\subsection{Performance Results for Complex Objects with Referentially Shared Subobjects}

In this section, we discuss the performance results for the case with accesses to foreign subobjects. We first discuss the performance when all subobjects that are accessed by a complexobject operation are foreign subobjects (i.e., subobjects that are physically clustered with other complex objects). In the discussed experiments, $f=10$ foreign subobjects were accessed per complex-object operation with update probability $u=20 \%$. We have also performed a sensitivity analysis of the $f$ parameter, by keeping the sum o+ $f$ (i.e., the total number of SO accesses per $\mathrm{CO}$ operation) constantly at 10 and varying f from 0 to 10 . In the following, we discuss to what extent foreign-subobject accesses changed the results obtained in Section 4.2. Strategy $\mathrm{S} 2$ is no longer considered here since it was always outperformed by S2/PS.

- Overall performance and lock conflicts:

As shown in Figure 14, the performance difference of S1 and S2/PS became even bigger, compared to the case without foreign-subobject accesses. For example, at a DMP of 12, S2/PS achieved 16 times higher throughput and 10 times shorter response time than $\$ 1$. As Figures $14 \mathrm{c}$ and $14 \mathrm{f} \mathrm{show,} \mathrm{this} \mathrm{performance} \mathrm{difference} \mathrm{is} \mathrm{mostly} \mathrm{caused} \mathrm{by} \mathrm{data} \mathrm{conten-}$ tion. For strategy $S 1$, both the total lock wait time and the conflict rate were substantially higher than in the experiment of Section 4.2. In addition, the number of deadlocks increased considerably.

With foreign-subobject accesses, the subobjects that are accessed by a subtransaction are scattered across the entire database. Compared to the results of Section 4.2, this fact destroyed the locality in the page accesses of a subtransaction. Thus, the total number of pages that are accessed within a transaction was increased, and the page access pattern was better randomized. For example, in the experiment of Section 4.2, the first SO access within each complex-object operation had a higher probability of getting blocked than the other $\mathrm{SO}$ accesses within the same $\mathrm{CO}$, as the latter benefit from the already acquired locks because of the high locality of subobject (and hence page) accesses. * Destroying this locality led to the disastrous performance of strategy $\mathrm{S} 1$.

- Log I/Os:

The most interesting aspect of the experiment with foreign-subobject accesses is the relationship between the DMP and the size of persistence spheres, as shown in Figure 14e. Whereas the average size of persistence spheres was not much affected by the DMP, the maximum persistence sphere size increased quite significantly with increasing DMP. As pointed out in Section 4.2, this effect can be quite beneficial, for it amounts to more batching of $\log 1 / O s$ (i.e., less but longer $\log 1 / O s$ ). However, batching $\log 1 / O s$ is desirable only up to a certain point. If persistence spheres become too large, then the writing of a persistence sphere adds a significant delay to the response time of the committing transaction that caused the $\log \mathrm{V} / \mathrm{O}$. In our experiments, the maximum persistence sphere at a DMP of 12 contained about 95 pages (each of size 2K). Writing this persistence sphere to a single log disk takes about 100 milliseconds, which is still negligible in our experiment but may be unacceptable in a different environment (e.g., with much faster CPUs).

* The net effect is similar to preclaiming, even though no preclaiming is actually performed. 


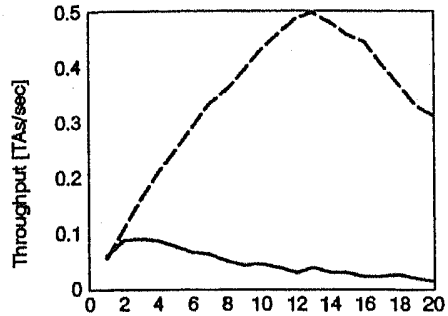

a) Throughput

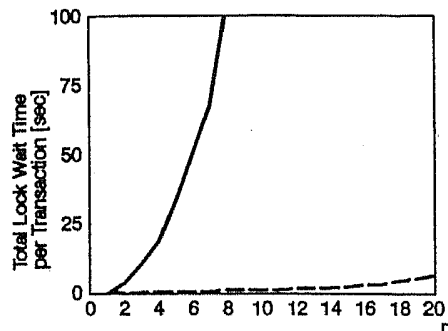

c) Total Lock Walt Time

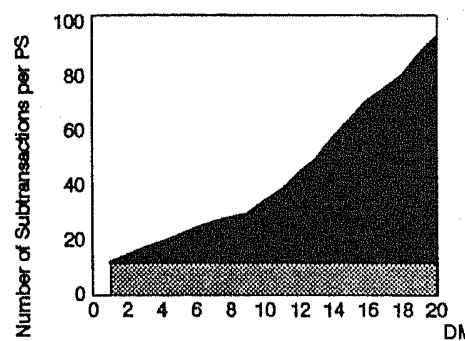

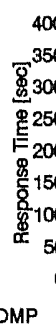

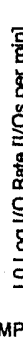

DMP

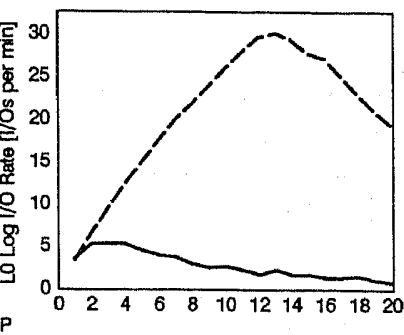

d) LO Log I/O Rate

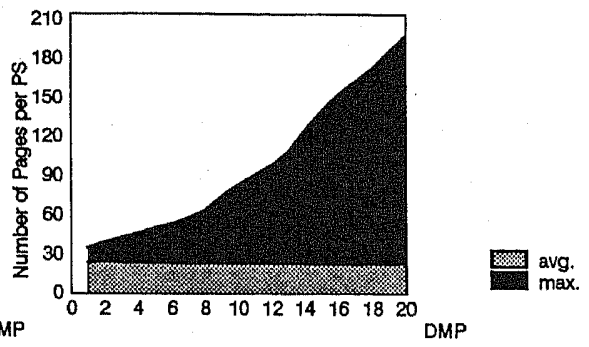

e) Persistence Sphere SIze
DMP

DMP
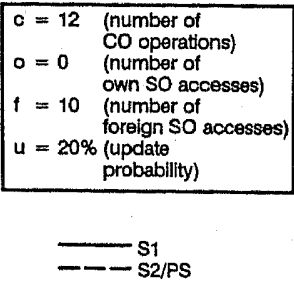

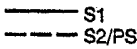

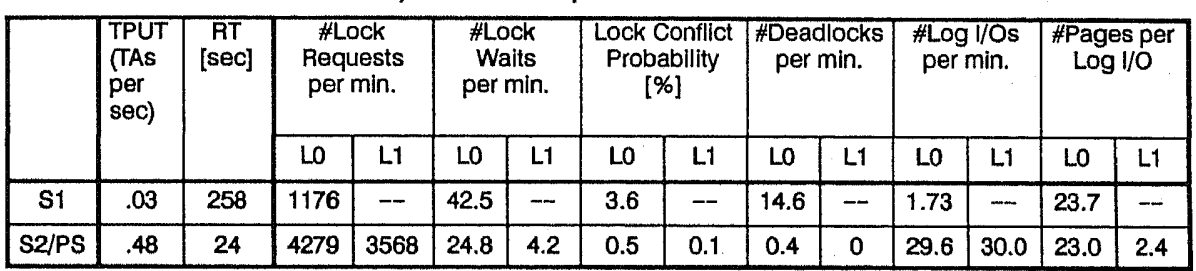

f) Performance Comparison at DMP 12

Fig. 14: Results of the Experiment with Foreign-Subobject Accesses $(f=10)$

Of course, writing the after-images in a persistence sphere is unavoidable in order to commit a transaction. In fact, our deferred write approach minimizes the number of pages that need to be written. The point, however, is that our method may cause unpredictable delays. The reason is that a large amount of log $1 / O$ work may be imposed on a transaction that has not done much work itself but happens to have a large persistence sphere constituted 
mostly by subtransactions of other active transactions. These unpredictable delays should be avoided in a high performance environment with response-time constraints. Note, however, that the delay caused by writing a large persistence sphere is still much shorter and therefore less severe than the delay that a synchronous checkpoint mechanism (e.g., [Gr81]) would cause.

There are two ways to eliminate or alleviate the described effect (none of which is currently implemented in DASDBS, though). The first way is to prevent the formation of large persistence spheres. This can be achieved by asynchronously writing persistence spheres whenever their size exceeds a certain threshold, even if the log $1 / O$ could be further deferred. Such a mechanism may actually increase the total amount of work since it may write more pages, but it has the advantage that it can distribute the log $1 / 0$ load more evenly over time. The second way to cope with large persistence spheres is to make their writing more efficient. This can be achieved by striping the log over multiple disks in a round-robin fashion (i.e., RAID-like striping) with a sufficiently large striping unit (e.g., a track). By exploiting the I/O parallelism of such a multi-disk log (cf. [SS90]), the response time penalty of the deferred write approach could be eliminated, even with much larger persistence spheres than we observed in our experiments.

Sensitivity of the number of foreign-subobject accesses:

The performance results with varying numbers of foreign-subobject accesses per complex-object operation are shown in Figure 15. These results essentially reconfirm the above observations. That is, with increasing number of foreign-subobject accesses, transactions loose locality which leads to more conflicts with $\mathrm{S1}$ and potentially larger persistence spheres with S2/PS.

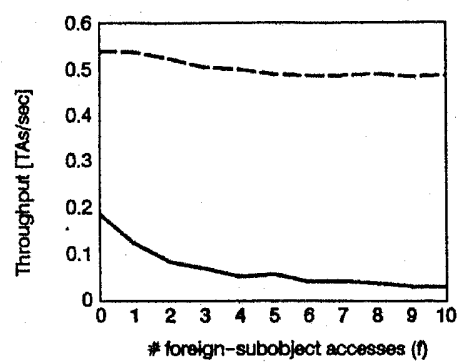

a) Throughput

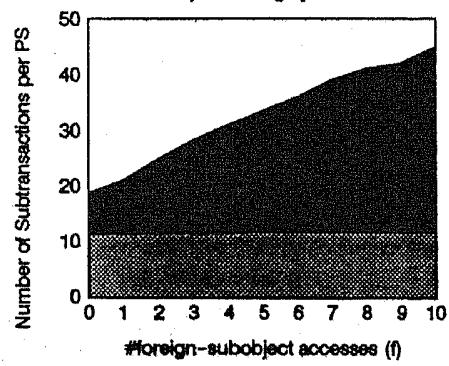

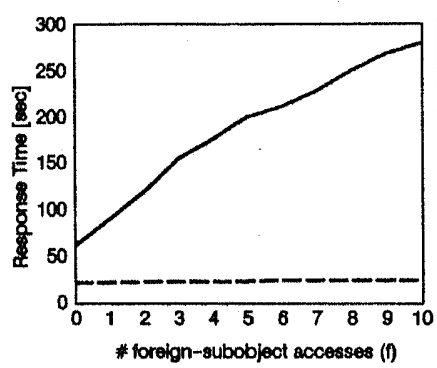

b) Response Time

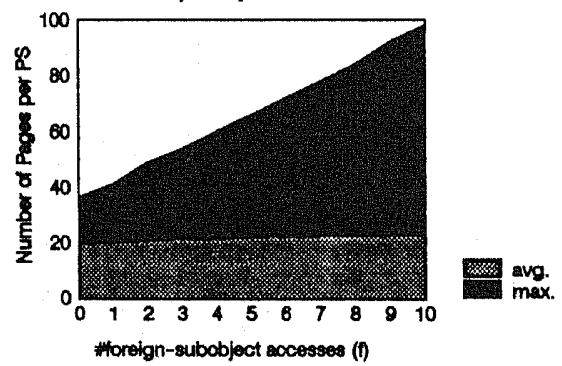

C) Persistence Sphere Size

Fig. 15: Sensitivity of the Number of Foreign-Subobject Accesses (f) 


\subsection{Preliminary Performance Results for Intra-Transaction Parallelism}

In a final series of experiments, we have started studying the impact of intra-transaction parallelism on multi-level transaction management. We concentrated on evaluating the strategy S2/PS since it always outperformed S2. ${ }^{*}$ In our benchmark, we assumed that all subtransactions of a transaction can indeed be executed in parallel; that is, there is no precedence order between the complex-object operations of a transaction. In the experiments, the effective degree of intra-transaction parallelism (DIP) was varied between 1 and 6 . For example, with a DIP of 6 , the first through sixth subtransaction of a transaction are executed in parallel, and subsequently the seventh through twelfth subtransaction are in parallel. We varied the DMP orthogonally to the DIP, in order to investigate how inter- and intra-transaction parallelism affect each other. Some preliminary results are discussed in the following.

- Overall performance:

Figures 16 and 17 show the performance results with and without foreign-subobject accesses, respectively. In the following, we concentrate on discussing the more interesting case with foreign-subobject accesses. The performance impact of the DIP turned out to be highly dependent on the DMP. With a low DMP, a relatively high DIP reduces the transaction response time and improves throughput; with a high DMP, however, the potential benefits of intra-transaction parallelism are clearly outweighted by the additional costs. The main bottleneck was the CPU capacity, as we had only 12 processors available but generated DMP * DIP processes with a CPU-intensive workload.

- Lock conflicts and latch conflicts:

As Figure 17c shows, the contention for locks, especially page locks at level LO, increased drastically with increasing values of the product DMP * DIP. For example, at DMP 12 and DIP 4, about $25 \%$ of a transaction's response time were spent waiting for a lock. This observation is remarkable as the same workload under the same strategy S2/PS showed almost no data contention in the previous experiments without intra-transaction parallelism even at a high DMP (see Figure 14). The phenomenon has two explanations. First, the execution time of a transaction increases considerabley with the product DMP * DIP, and therefore the potential for data contention increases. Second, intra-transaction parallelism increases the number of concurrently active subtransactions and hence the data contention at level LO. Thus, if the product DMP * DIP is not properly controlled, then short-term page locks become a performance-critical factor even though they are released at EOS.

Finally, the contention for internal latches became a severe performance problem at high values of DMP * DIP (see also Section 4.1). Even though this problem could be alleviated by tuning the code within the critical sections (which may include redesigning some of the buffer manager's and the lock manager's internal data structures), it cannot be completely eliminated if the number of concurrently active subtransactions is unrestricted.

These problems clearly show the need for load control for inter-and intra-transaction parallelism. We are pursuing an approach that dynamically adjusts the DMP and the DIP of the admitted transactions to the current load in terms of lock and latch contention as well as resource contention (cf. [CKL90, WHMZ90, MW91]).

- Log I/Os:

As far as the log $1 / O$ rate is concerned, the results with intra-transaction parallelism were no different from the results of Sections 4.2 and 4.3. That is, the number of $\mathrm{log} \mathrm{V} / \mathrm{Os}$ per time

\footnotetext{
* Note that intra-transaction parallelism requires some sort of subtransactions and is therefore not feasible with strategy $\mathbf{S 1}$ as it was implemented.
} 


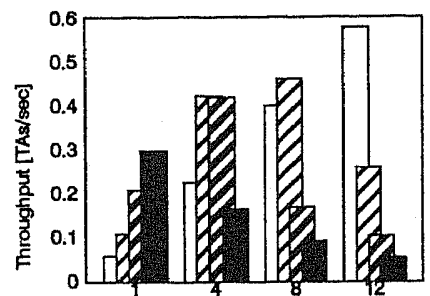

a) Throughput

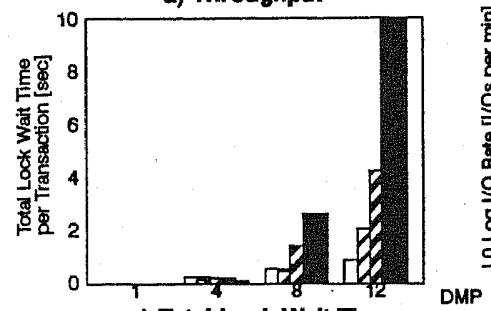

c) Total Lock Walt Time

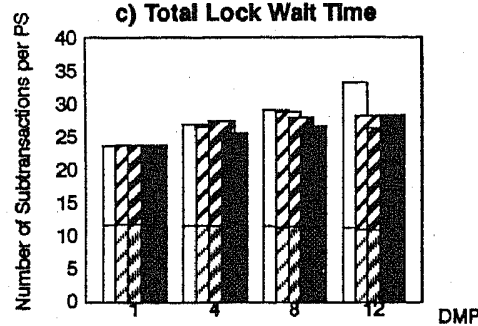

e) Persistence Sphere Slze (Average and Maximum)

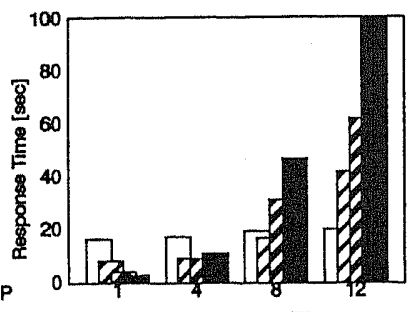

$\begin{array}{ll}0=12 & \text { (number of } \\ 0=10 & \text { (numberations) } \\ f=0 & \text { own So accesses) } \\ \text { (number of } \\ \text { foreign SO accesses) } \\ \mathrm{u}=20 \% & \begin{array}{l}\text { (update } \\ \text { probability) }\end{array} \\ & \end{array}$

b) Response TIme

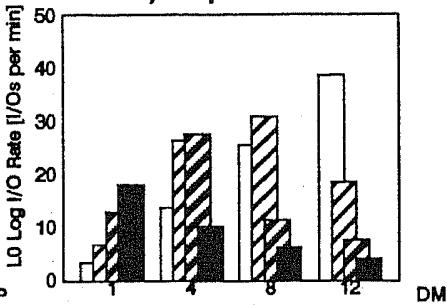

d) LO Log I/O Rate
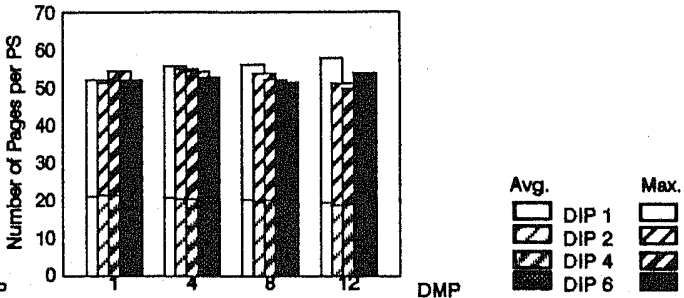

Fig. 16: Performance with Inter- and Intra-Transaction Parallelism for Disjoint Complex Objects

interval (see Figure 17d) was approximately proportional to the achieved transaction throughput.

We observed an interesting effect concerning the maximum size of persistence spheres at different DMP and DIP values. As Figure 17e shows, persistence spheres become larger with increasing DMP for all DIP values. The gradient of this increase, however, was smaller for high DIP values than for small ones. This may indicate that intra-transaction parallelism is beneficial for keeping persistence spheres small and thus making the execution time of the commit processing more predictable.

As an explanation of this phenomenon we offer the following hypothesis: The probability that two persistence spheres are merged increases with the product of the number of concurrently active subtransactions (i.e., DMP * DIP) and the average time between a subtransaction's EOS and the EOT of its transaction, or actually, with the integral of the number of completed but not yet forced subtransactions over time. The reason for this relationship is that a subtransaction is eligible for joining a persistence sphere only after its EOS, and is forced to the log file at EOT at the latest. 


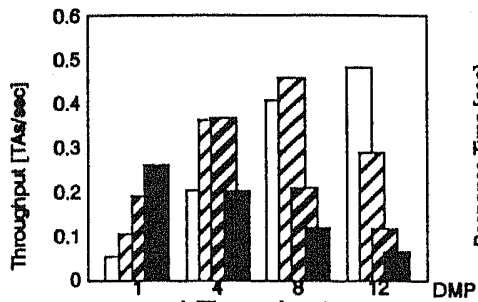

a) Throughput

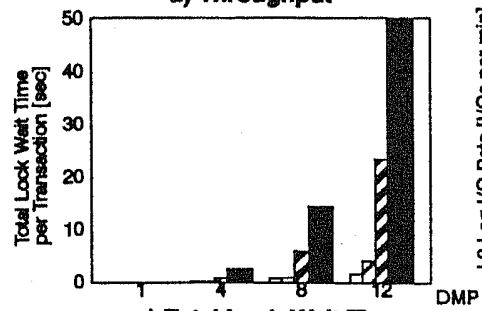

c) Total Lock Walt Time

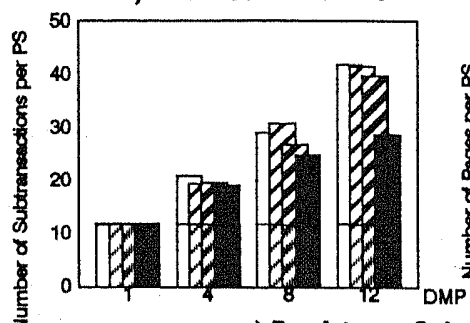

e) Persistence Sphere Size (Average and Maximum)

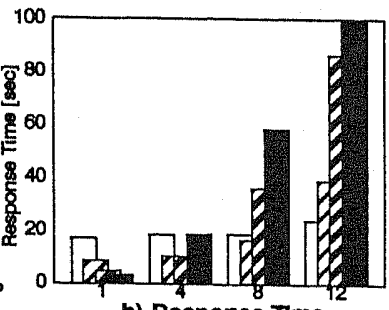

b) Response Time

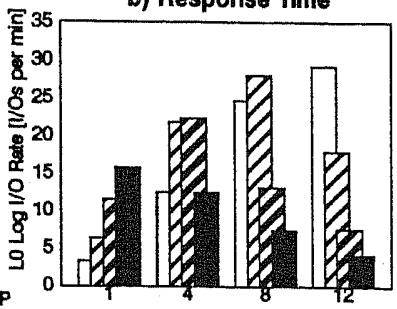

d) LO Log $1 / 0$ Rate

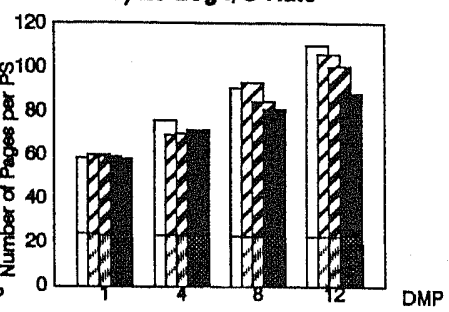

DMP

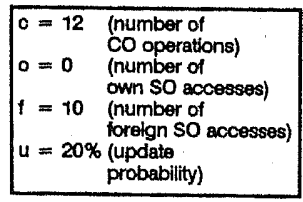

DMP

DIP DIP 2

DIP 4

DIP 6

Fig. 17: Performance with Inter- and Intra-Transaction Parallelism with Foreign-Subobject Accesses

Now, when we compare, for example, the case $D M P=12$ and $D I P=1$ with the case $D M P=2$ and $D I P=6$, the average time for which a subtransaction may join another transaction's persistence sphere can be estimated in a simplified way as follows. In the first case, the first subtransaction of a transaction consisting of 12 subtransactions stays for $\frac{11}{12}$ of the transaction's response time $\left(\mathrm{RT}_{1}\right)$ between EOS and EOT, the second subtransaction for $\frac{10}{12}$ of $R T_{1}$, and so on. This calculation yields an average of $\left(\left(\frac{11}{12}+\ldots+\frac{1}{12}\right){ }^{*} R T_{1}\right) / 12=\frac{11}{24} R T_{1}$ for the time interval during which a subtransaction may join a persistence sphere. In the second case, the first through sixth subtransaction of a transaction stay for $\frac{1}{2}$ of the transaction's response time $\left(R T_{2}\right)$ in the state between EOS and EOT; the seventh through twelfth subtransaction spend virtually no time between EOS and EOT if we assume ideal scheduling. This yields an average of $\left(\left(6 \cdot \frac{1}{2}\right) \cdot R T_{2}\right) / 12=\frac{R T_{2}}{4}$ for the critical time interval. Of course, this strawman calculation disregards lock wait time and scheduling effects. Nevertheless, we believe that it can be considered as an argument that intra-transaction parallelism is indeed beneficial for keeping (the variance of) persistence spheres small. 


\section{Comparison with Related Work}

Multi-level transaction management methods are implemented in the commercial database systems SQL/DS (which is essentially System R [Gr81]), Synapse [Ong84], and Informix-Turbo [Cu88]. These systems deal with transaction management at two levels: the record level and the page level. Their recovery methods use record-level redo, which slows down recovery at a warmstart; and they ensure the atomicity of record-level operations (including index updates) by periodically taking operation-consistent checkpoints that write all dirty pages back into the database. Such checkpoints adversely affect transaction response time, and become increasingly unacceptable with evergrowing buffer pool sizes.

An interesting unconventional multi-level recovery architecture has been implemented in the research prototype Kardamom [Bü88]. In this system, high-level update operations are performed on an object cache, and the propagation of updates onto pages is deferred until EOT. Thus, no high-level undo log records are needed, at the expense of performing redo at the obzct level. This approach may be well suited for a server-workstation environment where data is :xchanged at the object level (see also [lo89]). However, it does not become clear from the deicription of the algorithm if and how the approach can ensure the atomicity of high-level upJates that are propagated onto pages during a transaction's commit phase.

Our method of multi-level recovery is most closely related to the ARIES method [Mo89, ML89, MP91]. Even though the two methods were independently developed with very different design objectives, they have quite a few properties in common, as discussed in the following.

(C1) Both methods perform redo at the page level (i.e., "physical redo" in the terms of [Mo89]), thus minimizing the redo costs during a warmstart.

(C2) Both methods support semantic concurrency control in that they allow commutative update operations on the same object to be performed concurrently. In such a case, both methods consequently perform transaction undo by compensation rather than restoring previous object states.

(C3) As an unavoidable consequence of properties (C1) and (C2), both methods may have to redo updates of "loser transactions" that are afterwards undone by compensation during a warmstart. This principle is called the "repeating of history" paradigm in [Mo89].

(C4) To keep track of the modifications that are made by compensating (subtrans-) actions, both methods write a high-level log record when performing a compensating (subtrans-) action. These log records are called "compensation log records" (CLRs) in [M089].

Given these common properties, a simplified comparative view of our multi-level recovery method and the ARIES method is the following. Our method could "emulate" ARIES by 1) performing entry logging rather than after-image logging at the page level, 2) combining the L1 log and the LO log into a single physical log file, 3 ) adding a compensation backward chain between L1 log records to avoid undoing undo operations [Mo89], and 4) simply flushing all buffered log records whenever a persistence sphere has to be written. While the first three of these points would be (relatively simple) modifications or extensions of our method, the fourth point would actually be a simplification, at the expense of writing more log records (see below).

The similarity of ARIES and our method is especially remarkable because the two methods have been developed with very different design goals in mind. ARIES is an industrial-strength recovery method for relational DBMSs that is tailored to the prevalent storage structures of rela- 
tional systems. The multi-level recovery method, on the other hand, evolved from a theoretically well-founded but relatively puristic framework, aiming at high modularity and generality in that it can handle arbitrarily complex high-level operations. These different objectives have led to the following two important, subtle differences.

(D1) ARIES presumes that each high-level undo log record refers to exactly one page. This ensures the atomicity of high-level operations. The restriction is acceptable if one has only relational DBMS storage structures in mind, for a regular tuple always resides in one page, and page locking is considered to be good enough for long fields. The only significant problem arises with indexes, where a single high-level operation such as inserting a key may update multiple index pages because of index restructuring such as splits. In ARIES [ML89], such cases are dealt with by dividing a multi-page high-level update operation into a multi-page update that does not require (high-level) undo at all, and a single-page update that can be compensated.

In the example of inserting a new key that causes a split, the multi-page update is the split itself without inserting the key, and the atomic single-page update is the insertion of the new key into the restructured index. The atomicity of the multi-page update is in turn ensured by executing it as a "nested top action", which means that it is made persistent immediately upon its completion (and independently of the transaction to which itbelongs). This method entails that page-level undo and redo information is recorded during the operation's execution, but no high-level undo log record is written. Thus, the split operation cannot be undone once it is completed. While this is perfectly reasonable for index restructuring operations, bundling subtransaction atomicity together with persistence is clearly unacceptable for arbitrary multi-page update operations on complex objects. In our implementation of multi-level transaction management, ensuring subtransaction atomicity so that high-level compensation is feasible even for complex multi-page updates was one of the major challenges. It has been solved efficiently without sacrificing generality.

(D2) A less important yet remarkable difference between ARIES and our method is the amount of redo processing during a warmstart. Persistence spheres, as used in our method, are the minimal sets of redo log records that need to be written in order to ensure transaction persistence by page-level redo while observing subtransaction atomicity. ARIES, on the other hand, writes all generated log records to disk, which is much simpler. During the warmstart, ARIES therefore redoes all updates up to the point of the crash. The enhanced version called ARIES/RRH [MP91] avoids some of this redo work by checking, during the redo pass, if a redo log record of a loser transaction is followed by a redo log record of a winner transaction that refers to the same page. The update of the loser transaction need not be redone if (and, in ARIES/RRH, only if) this is not the case. In our method, such a check (which may even require look-ahead in the log [MP91]) is unnecessary because the critical redo log record would have been written to the log file only if the subtransaction that generated the log record were followed by a winner transaction that modified the critical page or if the dirty page were written back into the database before the crash occured.*

\footnotetext{
"We believe that this point is an interesting insight into the behavior of the two algorithms, but has probably only minor practical relevance. In addition, some of the ARIES considerations on repeating of history are motivated by media recovery issues (e.g., the desire to be able to perform medla recovery for individual pages). Our approach has disregarded media recovery, assuming that RAID-like fault tolerance techniques [PGK88, Gi89] can simplify media recovery to a large extent.
} 


\section{Further Performance Improvements}

The performance of our implementation, within the research prototype DASDBS, is encouraging despite an obvious lack of fine-tuning at the code level. Nevertheless, we are investigating various issues for improving the performance under specifically heavy load situations. These issues are briefly discussed in the following.

- "Light Weight" Subtransactions:

For particular types of high-level operations, the resulting reference pattern at the page level may have specific properties (e.g., pages are accessed in a specific order), so that it may be possible to guarantee deadlock-freedom between the corresponding subtransactions. Since the conflict rate at the page level is usually low, we may also simplify the queue management at virtually no risk of starvation. Under these conditions, it would be feasible to implement the page-level concurrency control between the eligible subtransaction types by latches rather than full-fledged locks. This would substantially reduce the CPU costs of multi-level concurrency control. Of course, the ultimate goal of such an approach would be to automatically generate the necessary latching protocol, based on the analysis of the possible page reference patterns of the particular types of subtransactions.

- Multi-Granularity Locking:

Another approach to reducing the CPU costs of multi-level concurrency control on complex objects is to incorporate multi-granularity locking at the object level. Unfortunately, while this is relatively simple and actually implemented in our system for the case of disjoint complex objects, it seems that the case of complex objects with referentially shared subobjects has not yet been completely solved (see [GK88, He90, HPS90]).

- Organization of Log Buffers and Log Files:

For subtransactions for which the LO log write can be deferred until EOT, it is not necessary to write the $L 1$ undo log records before the $L 0$ after-images because the $L O$ write is atomic. Thus, the transaction's $L 1$ log records could actually be discarded from the $L 1$ log buffer after the successful $L O \log$ write $\mathrm{I} / \mathrm{O}$. This would save $\log \mathrm{I} / \mathrm{O}$ s at the expense of having to change the L1 log buffer organization from a sequential ring buffer to a heap-like organization with direct addressing of log records. Note that the selective writing of after-images, which minimizes the amount of $L O$ redo log records (see Section 5), is also based on the fact that after-images are kept in the regular page buffer pool with directly addressable buffer frames rather than in a separate sequentially organized log buffer.

An orthogonal way of further reducing the amount of $\log \mathrm{l} / \mathrm{O}$ s could be to write the $L 1$ undo $\log$ records also into the $L O \log$ file, i.e., to combine the two logs into a single physical file. It seems that this could be done without major changes to the organization of the $\mathrm{LO}$ log file so that the efficient log compaction technique of [EB84] would still be applicable. The merging of the two logs would result in less (but slightly larger) set-oriented I/Os.

- Log File Partitioning:

Even though our measurements did not show a log $1 / 0$ bottleneck, the dramatically increasing speed of CPUs (due to RISC processors and/or multiprocessor systems) may eventually lead to a situation in which the transaction throughput is limited by the bandwidth of the (L1 or LO or combined) log disk. Such a bottleneck could only be eliminated by partitioning the log file(s) and distributing the partitions across multiple disks. This could be done transparently to the DBMS, by using RAIDs as a high-speed log device (cf. [SS90]), or by explicitly dealing with multiple log partitions. The latter approach has the potential advantage that, during a warmstart, the partitions of the log could be processed in parallel 
and independently (see [KHGP88] for similar considerations in a different context). Unlike previous approaches to parallel logging (e.g., [Ag85]), our method can indeed achieve this advantage by partitioning the L1 log by transaction numbers and the LO log by subtransaction numbers or page numbers.

Partitioning the LO log by subtransaction numbers is only feasible with after-image logging. In this case, we can use timestamps in the headers of after-images and apply the Thomas write rule (see, e.g., [BHG87]) to ensure that an after-image will not overwrite a more recent after-image of the same page during the parallel processing of the LO log partitions.

Partitioning the LO log by page numbers, on the other hand, leads to the problem that the after-images of a persistence sphere may be distributed across multiple partitions yet have to be written atomically. Rather than employing a full-fledged two-phase commit for this case, a cheaper solution could be to include in the header of each after-image an identification and the cardinality of the persistence sphere to which the after-image belongs. Then, during the parallel redo phase, we can check if a persistence sphere is complete or if the distributed $\log 1 / O$ failed on one of the partitions.

- Distributed Processing:

It is an open issue whether our approach to multi-level transaction management is appropriate also for distributed systems with data as well as function partitioning. For example, in a server-workstation environment, the object level could be implemented on the workstation and the page level on the server (cf. [DFMV90]). It is not clear how any of the recovery methods discussed in Section 5 would perform in such an environment.

\section{Conclusion}

The implemented method of multi-level transaction management has the following advantages.

- It allows exploiting the semantics of high-level operations to enhance concurrency.

- Our algorithms can deal with complex high-level operations on arbitrarily complex objects. In particular, it ensures the atomicity of high-level operations that modify multiple pages. This is a fundamental prerequisite for correctly dealing with compensation of high-level operations.

- These advantages are achieved at about the same log $1 / 0$ costs that an efficient page-oriented single-level recovery method has. Our method does not require a costly checkpoint mechanism, and it provides fast recovery after a crash.

- Our implementation supports also parallelism within a transaction.

The implemented system will serve as a testbed for further studies, especially on the tuning problems that arise with the coexistence of inter-and intra-transaction parallelism. This coexistence leads to more contention for resources (i.e., processors, memory, 1/O bandwidth, locks, latches), compared to a conventional database system with inter-transaction parallelism alone. Therefore, the decision on how much intra-transaction parallelism should be exploited in an individual transaction is dependent on the overall system load. Our long-term goal is to develop load control (i.e., transaction and subtransaction admission) and scheduling strategies that adjust the degree of inter-transaction parallelism and the degrees of intra-transaction parallelism of the individual transactions to the current load dynamically and automatically.

Another related problem that has not received much attention in the literature is how to adjust the parallel execution plan of a complex query to a situation in which the query's degree of (in- 
tra-transaction) parallelism is limited. The major tradeoff is how many processors (and other resources) should be used for function parallelism, that is, pipelining of basic operators, and how many processors should be used for data parallelism in the various operators of the query. The objective in addressing this problem is to develop tuning heuristics for parallel execution plans that incorporate both function and data parallelism.

These tuning problems are being addressed as part of the COMFORT project at ETH Zurich [WHMZ90] (see also [SSW90] for additional background). The ultimate goal of COMFORT is to automate tuning decisions for transaction processing in parallel database systems, thus simplifying the tricky job of system administrators and human tuning experts.

\section{Acknowledgements}

The method for deferred log writes and the concept of persistence spheres were designed jointly with Peter Brössler and Peter Muth. Their contribution is gratefully acknowledged. Further, we would like to thank the UBILAB of the Union Bank of Switzerland (Schweizerische Bankgesellschaft) and especially Prof. Rudolf Marty for supporting our work.

\section{References}

[Ag85] Agrawal, R., A Parallel Logging Algorithm for Multiprocessor Database Machines, 4th Int. Workshop on Database Machines, Springer, 1985

[An90] Anderson, T.L., Berre, A.H., Mallison, M., Porter, H., Schneider, B., The Hypermodel Benchmark, 2nd Int. Conf. on Extending Data Base Technology; Springer, 1990

[Bü88] von Bültzingsloewen, G., lochpe, C., Liedtke, R.-P., Dittrich, K.R., Lockemann, P.C., Two-Level Transaction Management in a Multiprocessor Database Machine, 3rd Int. Conf. on Data and Knowledge Bases, Jerusalem, 1988

[BBG89] Beeri, C., Bernstein, P.A., Goodman, N., A Model for Concurrency in Nested Transactions Systems, Journal of the ACM Vol.36 No.1, 1989

[BF89] Broessler, P., Freisleben, B., Transactions on Persistent Objects, Int. Workshop on Peristent Object Systems, Newcastle, Australia, 1989

BHG87] Bernstein, P.A., Hadzilacos, V., Goodman, N., Concurrency Control and Recovery in Jatabase Systems, Addison-Wesley, 1987

[BR90] Badrinath, B.R., Ramamritham, K., Performance Evaluation of Semantics-based Multilevel Concurrency Control Protocols, ACM SIGMOD Conf., 1990

[BS90] Buhr, P.A., Stroobosscher, R.A., The $\mu$ System: Providing Light-Weight Concurrency on Shared-Memory Multiprocessor Computers Running UNIX, Software - Practice and Experience Vol.20 No.9, 1990

[BSW88] Beeri, C., Schek, H.-J., Weikum, G., Multi-Level Transaction Management, Theoretical Art or Practical Need?, 1st Int. Conf. on Extending Database Technology, Venice, 1988, Springer, LNCS 303

[Cr84] Crus, Data Recovery in IBM Database 2, IBM Systems Journal Vol.23 No.2, 1984

[Cu88] Curtis, R.B., Informix-Turbo, IEEE COMPCON, 1988

[CF90] Cart, M., Ferrie, J., Integrating Concurrency Control into an Object-Oriented Database System, 2nd Int. Conf. on Extending Database Technology, Venice, 1990, Springer, LNCS 416 [CKL90] Carey, M.J., Krishnamurthi, S., Livny, M., Load Control for Locking: The 'Half-andHalf' Approach, ACM PODS Conf., 1990

[Du87] Duppel, N., Peinl, P., Reuter, A., Schiele, G., Zeller, H., Progress Report \#2 of PROSPECT, University of Stuttgart, 1987 
[DFMV90] DeWitt, D.J., Futtersack, P., Maier, D., Velez, F., A Study of Three Alternative Workstation-Server Architectures for Object Oriented Database Systems, VLDB Conf., 1990

[DG90] DeWitt, D.J., Gray, J., Parallel Database Systems, ACM SIGMOD Record Vol.19 No.4, 1990

[EB84] Elhardt, K., Bayer, R., A Database Cache for High Performance and Fast Restart in Database Systems, ACM TODS Vol.9 No.4, 1984

[EGLT76] Eswaran, K.P., Gray, J.N., Lorie, R.A., Traiger, I.L., The Notions of Consistency and Predicate Locks in a Database System, Communications of the ACM Vol.19 No.11, 1976

[FLMW88] Fekete, A., Lynch, N., Merritt, M., Weihl, W., Commutativity-Based Locking for Nested Transactions, Technical Report MIT/LCS/TM-370, MIT, Cambridge (Mass.), 1988, to appear in: Journal of Computer and System Sciences

[Ga83] Garcia-Molina, H., Using Semantic Knowledge for Transaction Processing in a Distributed Database, ACM TODS Vol.8 No.2, 1983

[Gi89] Gibson, G.A., Hellerstein, L., Karp, R.M., Katz, R.H., Patterson, D.A., Failure Correction Techniques for Large Disk Arrays, 3rd Int. Conf. on Architectural Support for Programming Languages and Operating Systems, 1989

[Gr78] Gray, J., Notes on Database Operating Systems, in: Operating Systems - An Advance Course, Springer, 1978

[Gr81] Gray, J., McJones, P., Blasgen, M., Lindsay, B., Lorie, R., Price, T., Putzolu, F., Traiger, I., The Recovery Manager of the System R Database Manager, ACM Computing Surveys Vol.13 No.2, 1981

[GK85] Gawlick, D., Kinkade, D., Varieties of Concurrency Control in IMS/VS Fast Path, IEEE Database Engineering Vol.8 No.2, 1985

[GK88] Garza, J., Kim, W., Transaction Management in an Object-Oriented Database System, ACM SIGMOD Conf., 1988

[GS87] Garcia-Molina, H., Salem, K., Sagas, ACM SIGMOD Conf., 1987

[GT90a] Graefe, G., Thakkar, S.S., Tuning a Parallel Database System on a Shared-Memory Multiprocessor, Technical Report, University of Colorado at Boulder, 1990

[GT90b] Graunke, G., Thakkar, S., Synchronization Algorithms for Shared-Memory Multiprocessors, IEEE Computer Vol.23 No.6, 1990

[Hä87] Härder, T., On Selected Performance Issues of Database Systems, 4th German Conf. on Performance Modeling of Computing Systems, Springer, 1987

[He87] Helland, P., Sammer, H., Lyon, J., Carr, R., Garrett, P., Reuter, A., Group Commit Timers and High Volume Transaction Systems, 2nd Int. Workshop on High Performance Transaction Systems, 1987, Springer, LNCS 359, 1989

[He90] Herrmann, U., Dadam, P., Küspert, K., Roman, E.A., Schlageter, G., A Lock Technique for Disjoint and Non-Disjoint Complex Objects, 2nd Int. Conf. on Extending Database Technology, Springer, 1990

[HH88] Hadzilacos, T., Hadzilacos, V., Transaction Synchronization in Object Bases, ACM PODS Conf., 1988

[HK89] Hudson, S.E., King, R., Cactis: A Self-Adaptive, Concurrent Implementation of an Object-Oriented Database Management System, ACM TODS Vol.14 No.3, 1989

[HR83] Härder, T., Reuter, A., Principles of Transaction-Oriented Database Recovery, ACM Computing Surveys Vol.15 No.4, 1983

[HPS90] Härder, T., Profit, M., Schöning, H., Supporting Parallelism in Engineering Databases by Nested Transactions, Technical Report, University of Kaiserslautern, 1990 
[HSS89] Haerder, T., Schoening, H., Sikeler, A., Parallel Query Evaluation: A New Approach to Complex Object Processing, IEEE Data Engineering Vol.12 No.1, 1989

[HW91] Hasse, C., Weikum, G., A Performance Evaluation of Multi-Level Transaction Management, VLDB Conf., 1991

[lo89] lochpe, C., Database Recovery in the Design Environment: Requirements Analysis and Performance Evaluation, Ph.D. Thesis, University of Karlsruhe, Germany, 1989

[KHGP88] King, R.P., Halim, N., Garcia-Molina, H., Polyzois, C.A., Management of a Remote Backup Copy for Disaster Recovery, IBM Research Report RC14291, Yorktown Heights, 1988 [KLS90] Korth, H.F., Levy, E., Silberschatz, A., Compensating Transactions: A New Recovery Paradigm, VLDB Conf., 1990

[Li79] Lindsay, B., et al., Notes on Distributed Databases, IBM Research Report RJ2571, San Jose, 1979

[Ma87] Martin, B.E., Modeling Concurrent Activities with Nested Objects, Int. Conf. on Distributed Computing Systems, Berlin, 1987

[M085] Moss, J.E.B., Nested Transactions: An Approach to Reliable Distributed Computing, MIT Press, 1985

[Mo89] Mohan, C., Haderle, D., Lindsay, B., Pirahesh, H., Schwarz, P., ARIES: A Transaction Recovery Method Supporting Fine-Granularity Locking and Partial Rollbacks Using WriteAhead Logging, IBM Research Report RJ6649, San Jose, 1989, to appear in: ACM TODS

[MGG86] Moss, J.E.B., Griffeth, N.D., Graham, M.H., Abstraction in Recovery Management, ACM SIGMOD Conf., 1986

[ML89] Mohan, C., Levine, F., ARIES/IM: An Efficient and High Concurrency Index Management Method Using Write-Ahead Logging, IBM Research Report RJ6846, San Jose, 1989

[MLC87]\} Moss, J.E.B., Leban, B., Chrysanthis, P.K., Finer Grained Concurrency for the Database Cache, IEEE Conf. on Data Engineering, 1987

[MP91] Mohan, C., Pirahesh, H., ARIES-RRH: Restricted Repeating of History in the ARIES Transaction Recovery Method, IEEE Conf. on Data Engineering, 1991

MR91] Muth, P., Rakow, T., Atomic Commitment for Integrated Database Systems, IEEE Conf. in Data Engineering, 1991

MS91] Murphy, M.C., Shan, M.-C., Execution Plan Balancing, IEEE Conf. on Data Engineerng, 1991

[MW91] Moenkeberg, A., Weikum, G., Conflict-driven Load Control for the Avoidance of DataContention Thrashing, IEEE Conf. on Data Engineering, 1991

[Ong84] Ong, K.S., Synapse Approach to Database Recovery, PODS Conf., 1984

[O'N86] O'Neil, P.E., The Escrow Transactional Method, ACM TODS Vol.11 No.4, 1986

[Pi90] Pirahesh, H., Mohan, C., Cheng, J., Liu, T.S., Selinger, P., Parallelism in Relational Data Base Systems: Architectural Issues and Design Approaches, 2nd Int. Symp. on Databases in Parallel and Distributed Systems, Dublin, 1990

[PGK88] Patterson, D.A., Gibson, G., Katz, R.H., A Case for Redundant Arrays of Inexpensive Disks (RAID), ACM SIGMOD Conf., 1988

[RGN90] Rakow, T.C., Gu, J., Neuhold, E.J., Serializability in Object-Oriented Database Systems, IEEE Conf. on Data Engineering, 1990

[Sh85] Shasha, D., What Good Are Concurrent Search Structure Algorithms for Databases Anyway?, IEEE Database Engineering Vol.8 No.2, 1985

[SDP91] Shrivastava, S.K., Dixon, G.N., Parrington, G.D., An Overview of the Arjuna Distributed Programming System, IEEE Software, January 1991 
[SG88] Shasha, D., Goodman, N., Concurrent Search Structure Algorithms, ACM TODS Vol.13 No.1, 1988

[SPSW90] Schek, H.-J., Paul, H.-B., Scholl, M.H., Weikum, G., The DASDBS Project: Objectives, Experiences, and Future Prospects, IEEE Transactions on Knowledge and Data Engineering Vol.2 No.1, 1990

[SS84] Schwarz, P.M., Spector, A.Z., Synchronizing Shared Abstract Types, ACM Transactions on Computer Systems Vol.2 No.3, 1984

[SS90] Seltzer, M., Stonebraker, M., Transaction Support in Read Optimized and Write Optimized File Systems, VLDB Conf., 1990

[SSW90] Schek, H.-J., Scholl, M.H., Weikum, G., From the Kernel to the coSMOS: The Database Research Group at ETH Zurich, Technical Report 136, Computer Science Dept., ETHZUrich, 1990

[SZ89] Skarra, A.H., Zdonik, S.B., Concurrency Control and Object-Oriented Databases, in: W. Kim, F.H. Lochovsky (eds.), Object-Oriented Concepts, Databases, and Applications, ACM Press, 1989

[We88] Weihl, W.E., Commutativity-Based Concurrency Control for Abstract Data Types, IEEE Transactions on Computers Vol.37 No.12, 1988

[We89] Weihl, W.E., The Impact of Recovery on Concurrency Control, ACM PODS Conf., 1989

Wei86] Weikum, G., A Theoretical Foundation of Multi-Level Concurrency Control, ACM PODS Conf., 1986

[Wei87] Weikum, G., Enhancing Concurrency in Layered Systems, Proc. 2nd Int. Workshop on High Performance Transaction Systems, 1987, Springer, LNCS 359, 1989

[Wei91] Weikum, G., Principles and Realization Strategies of Multilevel Transaction Management, ACM TODS Vol.16 No.1, 1991

[WHBM90] Weikum, G., Hasse, C., Broessler, P., Muth, P., Multi-Level Recovery, ACM PODS Conf., 1990

[WHMZ90] Weikum, G., Hasse, C., Mönkeberg, A., Zabback, P., The COMFORT Project: A Comfortable Way to Better Performance, Technical Report 137, Computer Science Dept., ETH Zurich, 1990

[WS84] Weikum, G., Schek, H.-J., Architectural Issues of Transaction Management in Layered Systems, VLDB Conf., 1984

[WS91] Weikum, G., Schek, H.-J., Multi-Level Transactions and Open Nested Transactions, IEEE Data Engineering Vol.14 No.1, 1991

Eidg. Techn. Hochschule Zürich Intormatikbiblicthek

ETH-ZEntium

$\mathrm{CH}-8092$ Zürich 
144 J.L. Marais

145 J. Mössenböck

146

H. E. Meier

147 G. Weikum, P. Zabback, P. Scheuermann

148

149

150 M.H. Scholl, Ch. Laasch

M. Tresch

151 C. Szyperski

152 M. Bronstein

153 G.H. Gonnet, D.W. Gruntz

154 G.H. Gonnet, St.A. Benner

155 D. Crippa

156 R. Griesemer

C. Pfister (ed.), B. Heeb,

J. Templ

157 T. Weibel, G. Gonnet

158 M. Scholl (ed.)

159 K. Gates

160 H. Mössenböck

161 S. Lalis
The GADGETS User Interface Management System

She: A Simple Hypertext Editor for Programs (vergriffen)

Schriftgestaltung mit Hilfe des Computers

Typographische Grundregein (vergriffen)

Dynamic File Allocation in Disk Arrays (vergriffen)

A New Linear Algorithm to Detect a Line Graph and Output its Root Graph

Conflict-Driven Load Control for the Avoidance of Data-Contention Thrashing

Updatable Views in Object-Oriented Databases (vergriffen)

Write - An extensible Text Editor for the Oberon System

On Solutions of Linear Ordinary Difierential Equations in their Coefficient Field

Algebraic Manipulation: Systems

Computational Biochemistry Research at ETH

A Special Case of the Dynamization Problem for Least Cost Paths

On the Linearization of Graphs and Writing Symbol Files

Oberon Technical Notes

An Algebra of Properties

Grundlagen von Datenbanken (Kurfassungen

des 3.GI-Workshops, Volkse, 21. - 24.5.91)

Using Inverse Iteration to Improve the Divide and Conquer Algorithm

Differences between Oberon and Oberon-2 The programming Language Oberon-2

XNet: Supporting Distributed Programming in the Oberon Environment 